\title{
Precise solution of few-body problems with stochastic variational method on correlated Gaussian basis
}

\author{
K. Varga ${ }^{1,2,3}$ and Y. Suzuki ${ }^{1}$ \\ ${ }^{1}$ Department of Physics, Niigata University, Niigata 950-21, Japan \\ ${ }^{2}$ RIKEN, Hirosawa, Wako, Saitama 351-01, Japan \\ and \\ ${ }^{3}$ Institute of Nuclear Research of the Hungarian Academy of Sciences, \\ Debrecen, H-4001, Hungary
}

(January 16, 2018)

\begin{abstract}
Precise variational solutions are given for problems involving diverse fermionic and bosonic $N=2-7$-body systems. The trial wave functions are chosen to be combinations of correlated Gaussians, which are constructed from products of the single-particle Gaussian wave packets through an integral transformation, thereby facilitating fully analytical calculations of the matrix elements. The nonlinear parameters of the trial function are chosen by a stochastic technique. The method has proved very efficient, virtually exact, and it seems feasible for any few-body bound-state problems emerging in nuclear or atomic physics.
\end{abstract}

PACS number(s): 21.45.+v, 21.10.Dr, 36.10.Dr, 02.60.Pn 


\section{INTRODUCTION}

Few-body problems of interacting particles have vital importance in all branches of physics from hadron to celestial levels. The main interest in the few-body problems lies in, e.g., finding an accurate solution for the system, testing the equation of motion and the conservation laws and symmetries, or looking for unknown interactions governing the system.

The investigation of few-nucleon systems interacting via realistic forces has always been in the center of the interest. Considerable effort has been exerted to obtain accurate groundstate properties of the few-nucleon systems with Faddeev-Yakubovsky (FY) [1 [3], variational [4 6], variational Monte Carlo (VMC) [7,8] and Green's function Monte Carlo (GFMC) methods [9, 10]. Most of these approaches has focused on three- or four-body problems.

To treat an $N$-particle system, one needs to cope with a large number of variables required to specify the wave function. By using $(N-1)$ relative coordinates to describe the system, for example, the discretization on a mesh with $p$ points, or the expansion of the function of the relative motion between the particles in terms of $p$ suitably chosen functions leads to $p^{(N-1)}$ mesh points or basis functions, which becomes prohibitively large with increasing $N$. All but the Monte Carlo methods face this difficulty as the number of particles increases. The VMC and GFMC methods have proved to be most successful by being able to go beyond the four-nucleon problem [1]1]. The secret of the efficiency of the Monte Carlo methods is the use of an importance sampling of the most relevant parts of the configuration space. This fact naturally raises a question: Even if the wave function of the $N$-particle system is expanded into an (excessively) large number of basis functions, can't one reduce the problem to a tractable one by selecting "the most important" basis functions ?

The aim of this paper is to present an alternative variational approach, the stochastic variational method (SVM) [12,13, by using the correlated Gaussians as basis functions 14,15]. Examples whose solutions were known before are used to demonstrate the performance of the method in treating nuclear as well as Coulomb interactions. To highlight some new physics, we have also included problems that have been hitherto unsolved. We give the formulation and some details of the method of the calculation and show applications to $N=2-7$-particle systems.

The variational foundation for the time-independent Schrödinger equation provides a solid and arbitrarily improvable framework for the solution of bound-state problems. The crucial point of the variational approach is the choice of the trial function. There are two widely applied strategies: (1) to select the most appropriate functional form to describe the short-range as well as long-range correlations and to compute the matrix elements by Monte Carlo technique, or (2) to use a number, possibly a great number, of simple terms, which facilitate the analytical calculation of the matrix elements. We follow the second course by using an expansion over a correlated Gaussian "basis".

To solve the $N$-particle problem, it is of prime importance to describe the correlation between the particles properly. The correlation is conveniently represented by a correlation factor, $F=\prod_{i<j}^{N} f_{i j}$ [4, [5, [7, 8, 16]. Most calculations have used this form of $F$ directly to evaluate the matrix elements. Such calculations are, however, fairly involved beyond the three-particle system and performed by Monte Carlo integrations. An alternative way

to incorporate the correlation is to approximate $f_{i j}$ as a linear combination of Gaussians 
$\exp \left(-\alpha_{i j}\left(\mathbf{r}_{i}-\mathbf{r}_{j}\right)^{2}\right)$. The $N$-particle basis function then contains product of these Gaussians: $\prod_{i<j}^{N} \exp \left(-\alpha_{i j}\left(\mathbf{r}_{i}-\mathbf{r}_{j}\right)^{2}\right)=\exp \left(-\sum_{i<j}^{N} \alpha_{i j}\left(\mathbf{r}_{i}-\mathbf{r}_{j}\right)^{2}\right)$. These Gaussian functions are widely used in variational calculations (see, for example, [15, 16]). We will apply a more general form of the correlated Gaussian functions which allow for nonzero orbital angular momentum, and will use the more convenient Jacobi relative coordinates instead of the relative distance vectors. The correlated Gaussians have an important advantage. Their Hamiltonian matrix elements can be analytically calculated in a unified framework, thus enabling one to avoid the formidable calculation involving the correlation factor $F$.

The variational approximation, however, may run into difficulties for the following reasons: (i) if the nonlinear parameters specifying the basis functions are varied, it is difficult to optimize them, (ii) if they are not, then the number of terms required may be excessively large, and, in both cases, (iii) the trial function of proper symmetry becomes extremely involved. For example, conventional methods [6, 15] for the choice of the Gaussian parameters lead to prohibitively large bases for more than 3 or 4 particles, which has limited the applicability of the Gaussian basis to few-body problems.

One can circumvent the optimization problem including large number of nonlinear parameters or the diagonalization of huge matrices by using the SVM. The SVM attempts to set up the most appropriate basis functions by the following stepwise procedure: One generates a would-be basis function by choosing the nonlinear parameters randomly, judges its utility by the energy gained by including it in the basis, and either keeps or discards it. One repeats this "trial and error" procedure until the basis set up leads to convergence. The original procedure of the SVM, proposed in [12], has recently been developed further and successfully applied to multicluster descriptions of light exotic nuclei, such as ${ }^{6} \mathrm{He}=\alpha+n+n$, ${ }^{8} \mathrm{He}=\alpha+n+n+n+n,{ }^{9} \mathrm{Li}=\alpha+t+n+n$, and ${ }^{9} \mathrm{C}=\alpha+{ }^{3} \mathrm{He}+p+p$ [13,17. Learning from these applications, we have now generalized and refined the method further to encompass diverse systems emerging in nuclear and atomic physics.

Besides the large number of nonlinear parameters, the treatment of the increasing number of partial waves in the expansion of the wave function would also pose a formidable task. We propose here an alternative formulation to cope with this problem. Instead of using the partial wave expansion, the angular dependence of the wave function is represented by a single solid spherical harmonics whose argument contains additional variational parameters. This form makes the calculation of the matrix elements for nonzero orbital angular momentum much simpler than other methods.

It will be demonstrated that the present method has several unique features: It is based on a fully analytical calculation for most types of interactions and thus ensures high accuracy and speed. Its calculational scheme is quite universal and needs no change depending on whether the system contains nuclear or Coulombic or other interactions. It has no difficulty in treating the system of particles of unequal masses. More importantly, the wave function is obtained in a compact, analytical form and thereby can be readily used in calculations of physical properties.

As you will see later, the present method has turned out to be very accurate, and we think it is worth while to make the method and the results easily available and reproducible for interested readers. We collect all the needed ingredients of our method in order. Some of the formulae are our original developments or generalizations of known relations to $N$-particle matrix elements, and some others are collected here to make the paper self-contained. The 
calculation of the matrix elements presented here is different from the one of Refs. [18,19] in many aspects: The motion of the centre-of-mass is removed from both the Hamiltonian and the wave function. Two-particle potential matrix elements of arbitrary radial form factor are evaluated in a unified way by reducing them to the calculation of appropriate correlation functions corresponding to the interaction. The calculation of the matrix elements is extended to nonzero orbital angular momentum as well. The symmetrization postulate is imposed on the wave function at the single-particle level, which provides several advantages, especially in evaluating the matrix elements of state-dependent realistic nuclear interactions.

The organization of the paper is as follows. Section II defines the correlated Gaussian basis functions and gives the details of the stochastic procedure of selecting the basis set. Section III contains the method of calculating the matrix elements. The main steps are the calculation of matrix elements in Slater determinants (or permanents for bosons) consisting

of single-particle Gaussian wave packets, the elimination of the center-of-mass motion with a very simple manipulation, and the transformation to the correlated Gaussian basis. This section also presents the modifications needed for treating systems of particles of unequal masses. Section IV presents numerical results for various systems of particles which interact via nuclear potentials or power-law potentials. Section $\mathrm{V}$ gives a brief summary. In the appendices the most important auxiliary formulae are collected to facilitate any future use of the formulation.

\section{THE CORRELATED GAUSSIANS AND THE STOCHASTIC VARIATIONAL METHOD}

\section{A. Basis functions}

Since the variational method is always limited by the form chosen as a trial function, the trial function must be flexible enough to be able to describe the full variety of correlations between the nucleons, e.g., the short-range correlation due to the strong repulsive force, the $\alpha$-clustering typical in some light nuclei, or the long-range correlation at large distances in light halo nuclei. The correlation between the nucleons can be described by functions of appropriate relative coordinates.

Any square-integrable function with angular momentum $l m$ can be approximated, to any desired accuracy, by a linear combination of nodeless harmonic-oscillator functions (Gaussians) of continuous size parameter $a$ :

$$
\Gamma_{l m}(\mathbf{r}) \sim \mathrm{e}^{-\frac{1}{2} a r^{2}} \mathcal{Y}_{l m}(\mathbf{r}), \quad \text { with } \quad \mathcal{Y}_{l m}(\mathbf{r})=r^{l} Y_{l m}(\hat{\mathbf{r}})
$$

A generalization of this to $N$-nucleon systems contains a product of the Gaussians as mentioned in the previous section. It is convenient to use a set of the Jacobi coordinates $\mathbf{x}=\left(\mathbf{x}_{1}, \ldots, \mathbf{x}_{N-1}\right)$, instead of $N(N-1) / 2$ relative distance vectors $\left(\mathbf{r}_{i}-\mathbf{r}_{j}\right)$. An $N$-nucleon basis function, a so-called correlated Gaussian, then looks like

$$
\psi_{(L S) J M T M_{T}}(\mathbf{x}, A)=\mathcal{A}\left\{\mathrm{e}^{-\frac{1}{2} \tilde{\mathbf{x}} A \mathbf{x}}\left[\theta_{L}(\mathbf{x}) \chi_{S}\right]_{J M} \mathcal{X}_{T M_{T}}\right\}
$$

where $\tilde{\mathbf{x}}$, the transpose of $\mathbf{x}$, stands for the row vector comprising the Jacobi coordinates. $\chi$ and $\mathcal{X}$ are the spin and isospin functions. $A$ is an $(N-1) \times(N-1)$ positive-definite, symmetric 
matrix of nonlinear parameters, specific to each basis element, and the quadratic form, $\tilde{\mathbf{x}} A \mathbf{x}$, involves scalar products of the Cartesian vectors:

$$
\tilde{\mathbf{x}} A \mathbf{x}=\sum_{i=1}^{N-1} \sum_{j=1}^{N-1} A_{i j} \mathbf{x}_{i} \cdot \mathbf{x}_{j}
$$

The operator $\mathcal{A}$ is an antisymmetrizer defined by

$$
\mathcal{A}=\frac{1}{\sqrt{N !}} \sum_{P}^{N !} \operatorname{sign}(P) P,
$$

where the sum runs over all permutations of the $N$ nucleon indices and $\operatorname{sign}(P)$ stands for the parity of the permutation $P$. For a system of identical bosons, the antisymmetrizer is to be replaced with a symmetrizer. For a general case $\mathcal{A}$ is to represent the operator that imposes the proper symmetry on the wave function.

The function $\theta_{L M_{L}}(\mathbf{x})$ in Eq. (2), which represents the angular part of the wave function, is a generalization of $\mathcal{Y}$ and can be chosen as a vector-coupled product of solid spherical harmonics of the Jacobi coordinates

$$
\theta_{L M_{L}}(\mathbf{x})=\left[\left[\left[\mathcal{Y}_{l_{1}}\left(\mathbf{x}_{1}\right) \mathcal{Y}_{l_{2}}\left(\mathbf{x}_{2}\right)\right]_{L_{12}} \mathcal{Y}_{l_{3}}\left(\mathbf{x}_{3}\right)\right]_{L_{123}}, \ldots\right]_{L M_{L}}
$$

Each relative motion has a definite angular momentum in Eq. (5). It may be important, however, to include several sets of angular momenta $\left(l_{1}, l_{2}, \ldots, l_{N-1} ; L_{12}, L_{123}, \ldots\right)$ for a realistic description. The various possible partial wave contributions increase the basis dimension; moreover, the calculation of matrix elements for this choice of $\theta_{L M_{L}}(\mathbf{x})$ becomes too complicated. This choice is apparently inconvenient especially as the number of nucleons increases. To avoid this, we propose a different choice as the generalization of $\mathcal{Y}$ :

$$
\theta_{L M_{L}}(\mathbf{x})=\eta_{K L M_{L}}(\mathbf{u}, \mathbf{x})=v^{2 K+L} Y_{L M_{L}}(\hat{\mathbf{v}}), \quad \text { with } \quad \mathbf{v}=\sum_{i=1}^{N-1} u_{i} \mathbf{x}_{i} .
$$

Only the total orbital angular momentum appears in this expression and it contains a parameter $\tilde{\mathbf{u}}=\left(u_{1}, \ldots, u_{N-1}\right)$. The vector $\mathbf{u}$ may be considered as a variational parameter and one may try to minimize the energy functional with respect to it. It defines a linear combination of the Jacobi coordinates, $\mathbf{v}$, and the wave function of the system is expanded in terms of its angle $\hat{\mathbf{v}}$. The minimization amounts to finding the most suitable angle or a linear combination of angles. The factor of $v^{2 K+L}$ plays an important role in improving the short-range behavior of the wave function. A remarkable advantage of this form of $\theta_{L M_{L}}(\mathbf{x})$ is that the calculation of matrix elements becomes much simpler than in the former case because the coupling of $(N-1)$ angular momenta is completely avoided.

The two forms of $\theta_{L M_{L}}(\mathbf{x})$ are in fact closely related to each other. Any of the functions of Eq. (5) may be expressed in terms of a linear combination of the terms, $v^{2 K+L} Y_{L M_{L}}(\hat{\mathbf{v}})$, by using some appropriate sets of $\mathbf{u}$ values provided that each term satisfies the condition $2 K+L \leq l_{1}+\cdots+l_{N-1}$ and contains a monomial of degree $l_{1}+\cdots+l_{N-1}-2 K-L$ in the variables, $\mathbf{x}_{1}{ }^{2}, \ldots, \mathbf{x}_{N-1}{ }^{2}$. Therefore, if one can calculate the matrix elements using $\theta_{L M_{L}}(\mathbf{x})$ defined in Eq. (6), then those with the previous form of $\theta_{L M_{L}}(\mathbf{x})$ can be obtained readily. 
The correlated Gaussian basis with the function $\theta_{L M_{L}}(\mathbf{x})$ of Eq. (6) has parity $(-1)^{L}$. To construct a function with parity $(-1)^{L+1}$, Eq. (6) must be slightly generalized, e.g., to

$$
\theta_{L M_{L}}(\mathbf{x})=\left[\eta_{K L}(\mathbf{u}, \mathbf{x}) \eta_{01}\left(\mathbf{u}^{\prime}, \mathbf{x}\right)\right]_{L M_{L}}
$$

To assure positive definiteness, the matrix $A$ in Eq. (2) is in general expressed as $A=$ $\tilde{G} A^{\prime} G$, where $G$ is an $(N-1) \times(N-1)$ orthogonal matrix containing $(N-1)(N-2) / 2$ parameters and $A^{\prime}$ is a diagonal matrix, $\left(A^{\prime}\right)_{i j}=a_{i}^{\prime} \delta_{i j}$, including $(N-1)$ positive parameters $a_{i}^{\prime}$. Although no restriction on the parameters of the matrix $G$ is in principle necessary, it is advisable to avoid too many variables if possible. The most naive choice would be to take $G$ as a unit matrix, which is equivalent to using only a single set of the Jacobi coordinates, and then to try to reach convergence by including higher partial waves successively. Many examples show [6,17], however, that this does not work well because the convergence is generally slow and moreover the computational cost of using high partial waves is quite expensive.

The matrix $G$ can also be chosen as one of the rotation matrices that connect the set of the Jacobi coordinates to other sets of independent relative coordinates. Figures 1a-1f show all topologically different sets of independent relative coordinates for a system of six identical particles. The set of coordinates in Fig. 1a is what we call the set of the Jacobi coordinates $\mathbf{x}$. A correlation conforming to a specific set of relative coordinates $\tilde{\mathbf{x}}^{\prime}=\left(\mathbf{x}_{1}^{\prime}, \ldots, \mathbf{x}_{N-1}^{\prime}\right)$ can be most efficiently described by tailoring the form of the basis function to this set of relative coordinates, that is, by using the form, $\exp \left\{-\frac{1}{2} \sum_{i=1}^{N-1} a_{i}^{\prime} \mathbf{x}_{i}^{\prime} \cdot \mathbf{x}_{i}^{\prime}\right\}$. Since the coordinates $\mathbf{x}^{\prime}$ can be obtained by an appropriate rotation $\mathcal{R}$ of the Jacobi coordinates as $\mathbf{x}^{\prime}=\mathcal{R} \mathbf{x}$, the basis function of such type can be clearly encompassed in the trial function of Eq. (2) by choosing $G=\mathcal{R}$. The correlated Gaussian basis thereby allows for various correlations between the nucleons and different asymptotics at large distances flexibly. Depending on the character of the problem a more general choice of $G$ might be necessary.

By selecting a set of basis functions $\left\{\psi_{i} ; i=1, \ldots, \mathcal{K}\right\}\left[\psi_{i} \equiv \psi_{\left(L_{i} S_{i}\right) J M T M_{T}}\left(\mathbf{x}, A_{i}\right)\right]$ that adequately spans the state space, the wave function of the $N$-nucleon system can be expanded as

$$
\Psi=\sum_{i=1}^{\mathcal{K}} c_{i} \psi_{i},
$$

where $\tilde{\mathbf{c}}=\left(c_{1}, \ldots, c_{\mathcal{K}}\right)$ is the set of linear variational parameters. The Ritz variational method defined by this trial function reduces to the generalized algebraic eigenvalue problem

$$
\mathcal{H} \mathbf{c}=E \mathcal{N} \mathbf{c}
$$

where $\mathcal{H}$ and $\mathcal{N}$ are, respectively, the matrices of the Hamiltonian and of the overlap

$$
\mathcal{H}_{i j}=\left\langle\psi_{i}|H| \psi_{j}\right\rangle, \quad \text { and } \quad \mathcal{N}_{i j}=\left\langle\psi_{i} \mid \psi_{j}\right\rangle \quad(i, j=1, \ldots, \mathcal{K}) .
$$

\section{B. Stochastic selection of parameters and solution of the eigenvalue problem}

Since a linear combination of the correlated Gaussians forms a dense set, there are different sets of $A$ that represent the wave function equally well. This enables one to select 
the most appropriate parameters randomly. We set up the basis stepwise by choosing $A$ from a preset domain of the parameter space and increase the basis dimension one by one. In the first step we select a number of parameter sets $A$ randomly, and keep the one that gives the lowest energy. Next we generate a new random set and calculate the energy with this two-element basis. As one more basis state always lowers the energy, we quantify its "utility" by the energy gained by including it in the basis. If the energy gain is larger than a preset value, $\epsilon$, then we admit this state to the basis, otherwise we discard it and try a new random candidate. This is repeated until the energy converges. The rate of convergence can be controlled by dynamically decreasing the value of $\epsilon$ during the search. This procedure is more advantageous than the earlier versions [12,13 and, although not a full optimization, results in very good and relatively small bases. A similar procedure, called "stochastic diagonalization" has been used to determine the smallest eigenvalue of extremely large matrices [20].

To have an economical algorithm for setting up the basis by a trial and error method, one has to find an efficient way to solve the eigenvalue problem, Eq. (9). The full diagonalization is rather time consuming and in fact unnecessary because (i) in the $(\mathcal{K}+1)$ th step of the procedure we can use the result of the $\mathcal{K}$ th step and (ii) to judge the usefulness of a wouldbe basis state, only the lowest eigenvalue is needed. Let us assume that in the $\mathcal{K}$ th step the Hamiltonian matrix $\mathcal{H}$ is diagonalized; its eigenenergies are $E_{1} \leq E_{2} \leq \ldots \leq E_{\mathcal{K}}$ and its corresponding normalized eigenfunctions are $\Psi_{1}, \ldots, \Psi_{\mathcal{K}}$. The eigenvalue problem in the $(\mathcal{K}+1)$ th step takes the form

$$
\begin{gathered}
\left(\begin{array}{ccccc}
E_{1} & 0 & \ldots & 0 & \left\langle\Psi_{1}|H| \psi_{\mathcal{K}+1}\right\rangle \\
0 & & & & \\
\vdots & & & \vdots & \vdots \\
0 & \ldots & E_{\mathcal{K}} & \left\langle\Psi_{\mathcal{K}}|H| \psi_{\mathcal{K}+1}\right\rangle \\
\left\langle\psi_{\mathcal{K}+1}|H| \Psi_{1}\right\rangle & \ldots & \left\langle\psi_{\mathcal{K}+1}|H| \Psi_{\mathcal{K}}\right\rangle & \left\langle\psi_{\mathcal{K}+1}|H| \psi_{\mathcal{K}+1}\right\rangle
\end{array}\right)\left(\begin{array}{c}
c_{1} \\
\vdots \\
\vdots \\
c_{\mathcal{K}} \\
c_{\mathcal{K}+1}
\end{array}\right) \\
=E\left(\begin{array}{ccccc}
c_{1} \\
\vdots \\
0 & 0 & \ldots & 0 & \left\langle\Psi_{1} \mid \psi_{\mathcal{K}+1}\right\rangle \\
\vdots & & & \vdots & \vdots \\
0 & \ldots & 1 & \left\langle\Psi_{\mathcal{K}} \mid \psi_{\mathcal{K}+1}\right\rangle \\
\left\langle\psi_{\mathcal{K}+1} \mid \Psi_{1}\right\rangle & \ldots & \left\langle\psi_{\mathcal{K}+1} \mid \Psi_{\mathcal{K}}\right\rangle & \left\langle\psi_{\mathcal{K}+1} \mid \psi_{\mathcal{K}+1}\right\rangle
\end{array}\right)\left(\begin{array}{c} 
\\
c_{\mathcal{K}} \\
c_{\mathcal{K}+1}
\end{array}\right) .
\end{gathered}
$$

By using the Gram-Schmidt orthogonalization method, that is, by defining

$$
\left|\bar{\psi}_{\mathcal{K}+1}\right\rangle=\frac{\left|\psi_{\mathcal{K}+1}\right\rangle-\sum_{i=1}^{\mathcal{K}}\left|\Psi_{i}\right\rangle\left\langle\Psi_{i} \mid \psi_{\mathcal{K}+1}\right\rangle}{\left(\left\langle\psi_{\mathcal{K}+1} \mid \psi_{\mathcal{K}+1}\right\rangle-\sum_{i=1}^{\mathcal{K}}\left\langle\psi_{\mathcal{K}+1} \mid \Psi_{i}\right\rangle\left\langle\Psi_{i} \mid \psi_{\mathcal{K}+1}\right\rangle\right)^{1 / 2}}
$$

this generalized eigenvalue equation can be reduced to the conventional form

$$
\left(\begin{array}{ccccc}
E_{1} & 0 & \ldots & 0 & q_{1} \\
0 & & & \\
\vdots & & & \vdots \\
0 & \ldots & E_{\mathcal{K}} & q_{\mathcal{K}} \\
q_{1} & \ldots & q_{\mathcal{K}} & a
\end{array}\right)\left(\begin{array}{c}
c_{1} \\
\vdots \\
\vdots \\
c_{\mathcal{K}} \\
c_{\mathcal{K}+1}
\end{array}\right)=E\left(\begin{array}{c}
c_{1} \\
\vdots \\
\vdots \\
c_{\mathcal{K}} \\
c_{\mathcal{K}+1}
\end{array}\right)
$$


where

$$
q_{i}=\left\langle\Psi_{i}|H| \bar{\psi}_{\mathcal{K}+1}\right\rangle, \quad a=\left\langle\bar{\psi}_{\mathcal{K}+1}|H| \bar{\psi}_{\mathcal{K}+1}\right\rangle
$$

The eigenvalues are easily obtained by finding the roots of the secular equation

$$
\lambda(E) \equiv \prod_{i=1}^{\mathcal{K}}\left(E_{\mathcal{K}}-E\right)\left((a-E)-\sum_{j=1}^{\mathcal{K}} \frac{q_{j}^{2}}{E_{j}-E}\right)=0 .
$$

This secular equation has $(\mathcal{K}+1)$ roots $\left\{E^{\prime}{ }_{i} ; i=1, \ldots, \mathcal{K}+1\right\}$ fulfilling the inequalities $E^{\prime}{ }_{1} \leq$ $E_{1} \leq E_{2}^{\prime} \leq E_{2} \leq \ldots \leq E_{\mathcal{K}} \leq E^{\prime} \mathcal{K}+1$. The eigenvectors are readily obtained after substituting the eigenvalues $\left\{E^{\prime}{ }_{i} ; i=1, \ldots, \mathcal{K}+1\right\}$ into Eq. (13). Note that one has to determine only the lowest eigenvalue $E^{\prime}{ }_{1}$ for the admittance criterion.

\section{CALCULATION OF THE MATRIX ELEMENTS}

In this section we will give the details of the method of calculating the matrix elements between the basis function of Eq. (2). The calculation consists of three steps: (A)The calculation of the matrix elements between the Slater determinants of the Gaussian wavepacket single-particle functions, (B)A transformation from the single-particle coordinate representation to the relative and center-of-mass coordinate representation, (C)An integral transformation from the Gaussian wave-packet functions to the correlated Gaussian basis. A procedure similar to steps (A) and (B) was used to manipulate algebraically the antisymmetrization operation and the transformation of the coordinates for complex cluster systems [21]. In step (A) the Slater determinant for the $N$-nucleon wave function is constructed by distributing the nucleons at positions $\left(\mathbf{s}_{1}, \ldots, \mathbf{s}_{N}\right)$. These position vectors serve as the generator coordinates. The Slater determinant of the Gaussian wave packets is often used in nuclear theory, e.g., in cluster model 21 24] and fermionic or antisymmetrized molecular dynamics [25,26]. The Hamiltonian matrix elements are analytically evaluated with the use

of technique of the Slater determinants [27,22], and can be expressed as a function of the generator coordinates. In step (B) the center-of-mass motion is completely separated from the intrinsic motion, and thus the trial wave function acquires the translational invariance. The separation of the center-of-mass motion is particularly simple in this formulation. In the last step (C) the matrix elements expressed in terms of the intrinsic generator coordinates are transformed to those between the correlated Gaussian basis functions with a definite angular momentum. Some of the essential parts of the calculational scheme is our original development, and some of them is a generalization of the technique used in the nuclear cluster model (see, for example, [28]). We also show in subsection III.D those modifications which are needed to treat the system of particles of unequal masses.

\section{A. Slater determinants of Gaussian wave packets}

The $i$ th nucleon with mass $m$, spin $\sigma_{i}$ and isospin $\tau_{i}$ is to be put in the single-particle Gaussian wave packet 


$$
\hat{\varphi}_{\mathbf{s}_{i} \sigma_{i} \tau_{i}}^{\nu}\left(\mathbf{r}_{i}\right)=\varphi_{\mathbf{s}_{i}}^{\nu}\left(\mathbf{r}_{i}\right) \chi_{\frac{1}{2} \sigma_{i}} \mathcal{X}_{\frac{1}{2} \tau_{i}}
$$

with

$$
\varphi_{\mathbf{s}_{i}}^{\nu}\left(\mathbf{r}_{i}\right)=\left(\frac{2 \nu}{\pi}\right)^{3 / 4} \mathrm{e}^{-\nu\left(\mathbf{r}_{i}-\mathbf{s}_{i}\right)^{2}}, \quad \text { and } \quad \nu=\frac{m \omega}{2 \hbar}
$$

where $\mathbf{r}_{i}$ is the position vector of the nucleon, $\chi_{\frac{1}{2} \sigma_{i}}$ and $\mathcal{X}_{\frac{1}{2} \tau_{i}}$ are its spin and isospin function. The angular frequency $\omega$ is not a variational parameter and may be taken an arbitrary constant. The $\mathbf{s}_{i}$ parameter or "generator" coordinate will be used in an integral transformation to derive the matrix elements between the Gaussian basis functions. A Slater determinant of these Gaussian packets is defined by

$$
\phi_{\kappa}\left(\mathbf{s}_{1}, \ldots, \mathbf{s}_{N}\right)=\mathcal{A}\left\{\prod_{i=1}^{N} \hat{\varphi}_{\mathbf{s}_{i} \sigma_{i} \tau_{i}}^{\nu}\left(\mathbf{r}_{i}\right)\right\},
$$

where $\kappa=\left(\sigma_{1} \tau_{1}, \ldots, \sigma_{N} \tau_{N}\right)$ is the set of the spin-isospin quantum numbers of the nucleons. The spins and the isospins of the nucleons are successively coupled to add up, respectively, to the total spin $S M_{S}$ and isospin $T M_{T}$ of the $N$-nucleon system:

$$
\chi_{S M_{S}}=\left[\left[\left[\chi_{\frac{1}{2}} \chi_{\frac{1}{2}}\right]_{S_{12}} \chi_{\frac{1}{2}}\right]_{S_{123}}, \ldots\right]_{S M_{S}}, \quad \mathcal{X}_{T M_{T}}=\left[\left[\left[\mathcal{X}_{\frac{1}{2}} \mathcal{X}_{\frac{1}{2}}\right]_{T_{12}} \mathcal{X}_{\frac{1}{2}}\right]_{T_{123}}, \ldots\right]_{T M_{T}}
$$

To simplify the notation, the intermediate quantum numbers are suppressed in the following. The wave function in the "generator coordinate space" with the definite spin and isospin quantum numbers is a linear combination of the Slater determinants of the Gaussian packets:

$$
\Phi_{S M_{S} T T_{z}}\left(\mathbf{s}_{1}, \ldots, \mathbf{s}_{N}\right)=\mathcal{A}\left\{\varphi_{\mathbf{s}_{1}}^{\nu}\left(\mathbf{r}_{1}\right) \ldots \varphi_{\mathbf{s}_{N}}^{\nu}\left(\mathbf{r}_{N}\right) \chi_{S M_{S}} \mathcal{X}_{T M_{T}}\right\}=\sum_{\kappa} c_{\kappa} \phi_{\kappa}\left(\mathbf{s}_{1}, \ldots, \mathbf{s}_{N}\right)
$$

where $c_{\kappa}$ is a product of the Clebsch-Gordan coefficients needed to couple the spin and isospin as defined in Eq. (19).

The Hamiltonian of the $N$-nucleon system reads as

$$
H=\sum_{i=1}^{N} \frac{\mathbf{p}_{i}^{2}}{2 m}+\sum_{i<j}^{N} V_{i j} .
$$

The matrix elements of the Slater determinants can easily be evaluated using the well-known rules [27,22]. To make this paper self-contained, we have collected all the needed ingredients in Appendices A, B and C. The overlap of the Slater determinants is found to take the form

$$
\left\langle\Phi_{S M_{S} T M_{T}}\left(\mathbf{s}_{1}, \ldots, \mathbf{s}_{N}\right) \mid \Phi_{S M_{S} T M_{T}}\left(\mathbf{s}^{\prime}{ }_{1}, \ldots, \mathbf{s}^{\prime}{ }_{N}\right)\right\rangle=\sum_{i=1}^{n_{o}} C_{i}^{(o)} \mathrm{e}^{-\frac{1}{2} \tilde{\mathbf{s}} A_{i}^{(o)} \mathbf{s}}
$$

where $A_{i}^{(o)}$ is a $2 N \times 2 N$ real, symmetric matrix and $\tilde{\mathbf{s}}$ stands for the $2 N$-dimensional row vector comprising the single-particle generator coordinates, $\left(\mathbf{s}_{1}, \ldots, \mathbf{s}_{N}, \mathbf{s}_{1}^{\prime}, \ldots, \mathbf{s}_{N}^{\prime}\right)$. To simplify the notation, we refer to the set of the vectors $\left(\mathbf{s}_{1}^{\prime}, \ldots, \mathbf{s}_{N}^{\prime}\right)$ alternatively as $\left(\mathbf{s}_{N+1}, \ldots, \mathbf{s}_{2 N}\right)$. Note, therefore, that the quadratic form, $\tilde{\mathbf{s}} A_{i}^{(o)} \mathbf{s}$, reads as 


$$
\tilde{\mathbf{s}} A_{i}^{(o)} \mathbf{s}=\sum_{j=1}^{2 N} \sum_{k=1}^{2 N}\left(A_{i}^{(o)}\right)_{j k} \mathbf{s}_{j} \cdot \mathbf{s}_{k} .
$$

The matrix elements of the kinetic energy operator can also be expressed in terms of the same $C_{i}^{(o)}$ 's and $A_{i}^{(o)}$ 's as

$$
\begin{aligned}
& \left\langle\Phi_{S M_{S} T M_{T}}\left(\mathbf{s}_{1}, \ldots, \mathbf{s}_{N}\right)\left|\sum_{i=1}^{N} \frac{\mathbf{p}_{i}^{2}}{2 m}\right| \Phi_{S M_{S} T M_{T}}\left(\mathbf{s}_{1}^{\prime}, \ldots, \mathbf{s}_{N}^{\prime}\right)\right\rangle \\
& =\frac{\hbar \omega}{2} \sum_{i}^{n_{o}} C_{i}^{(o)}\left(\frac{3}{2} N-\frac{1}{2} \tilde{\mathbf{s}} A_{i}^{(o)} \mathbf{s}\right) \mathrm{e}^{-\frac{1}{2} \tilde{\mathbf{s}} A_{i}^{(o)} \mathbf{s}} .
\end{aligned}
$$

The matrix elements of any term of the two-body interaction can be expressed as an integral of the two-particle correlation function multiplied by the radial form factor, $V(r)$, of the term $V_{i j}$ as below:

$$
\begin{aligned}
& \left\langle\Phi_{S M_{S} T M_{T}}\left(\mathbf{s}_{1}, \ldots, \mathbf{s}_{N}\right)\left|\sum_{i<j}^{N} V_{i j}\right| \Phi_{S^{\prime} M_{S}^{\prime} T^{\prime} M_{T}^{\prime}}\left(\mathbf{s}_{1}^{\prime}, \ldots, \mathbf{s}_{N}^{\prime}\right)\right\rangle \\
& =\int d \mathbf{r} V(r) \mathrm{e}^{-\nu r^{2}} \sum_{i=1}^{n_{p}} C_{i}^{(p)} P_{i}(\mathbf{s}, \mathbf{r}) \mathrm{e}^{-\frac{1}{2} \tilde{\mathbf{s}} A_{i}^{(p)} \mathbf{s}+\mathbf{d}_{i} \cdot \mathbf{r}}
\end{aligned}
$$

where $P_{i}(\mathbf{s}, \mathbf{r})$ is a polynomial of $\mathbf{s}$ and $\mathbf{r}, A_{i}^{(p)}$ a $2 N \times 2 N$ symmetric matrix, and $\mathbf{d}_{i} \cdot \mathbf{r}$ takes the form

$$
\mathbf{d}_{i} \cdot \mathbf{r}=\sum_{j=1}^{2 N} \mathcal{D}_{(i) j} \mathbf{s}_{j} \cdot \mathbf{r}
$$

The polynomial part reduces to unity $\left(P_{i}(\mathbf{s}, \mathbf{r})=1\right)$ in the case of pure central forces, but it has a rather simple form for spin-orbit, tensor, and other interactions as well. See Appendices B and C. The $C_{i}^{(o)}$ 's, $A_{i}^{(o)}$ 's, $C_{i}^{(p)}$ 's, and $A_{i}^{(p)}$ 's, etc. are obtained with the use of mathematical manipulation languages or fortran programs. See Appendix C.

\section{B. Transformation to relative and center-of-mass coordinates}

To eliminate the center-of-mass motion, we transform the single-particle coordinates to the relative and center-of-mass coordinates. For this purpose we choose one particular set of relative coordinates, the Jacobi coordinates, which is expressed in terms of the single-particle coordinates $\mathbf{r}_{i}$ as

$$
\mathbf{x}_{i}=\sum_{k=1}^{N} U_{i k} \mathbf{r}_{k} \quad(i=1, \ldots, N)
$$

where the transformation matrix $U$ is defined by 


$$
U=\left(\begin{array}{ccccc}
-1 & 1 & 0 & \ldots & 0 \\
-\frac{1}{2} & -\frac{1}{2} & 1 & \ldots & 0 \\
\vdots & & & & \vdots \\
-\frac{1}{N-1} & -\frac{1}{N-1} & \ldots & \ldots & 1 \\
\frac{1}{N} & \frac{1}{N} & \ldots & \ldots & \frac{1}{N}
\end{array}\right), \quad \text { and } \quad U^{-1}=\left(\begin{array}{ccccc}
-\frac{1}{2} & -\frac{1}{3} & \ldots & -\frac{1}{N} & 1 \\
\frac{1}{2} & -\frac{1}{3} & -\frac{1}{N} & 1 \\
0 & \frac{2}{3} & \vdots & \vdots \\
\vdots & \vdots & & \vdots & \vdots \\
0 & 0 & \ldots & \frac{N-1}{N} & 1
\end{array}\right)
$$

Similarly, the single-particle generator coordinates are transformed to the relative and centerof-mass generator coordinates:

$$
\mathbf{S}_{i}=\sum_{k=1}^{N} U_{i k} \mathbf{s}_{k}, \quad \mathbf{S}_{i}^{\prime}=\sum_{k=1}^{N} U_{i k} \mathbf{s}_{k}^{\prime} \quad(i=1, \ldots, N) .
$$

The reduced masses corresponding to the transformation $U$ are given by

$$
\mu_{i}=\frac{i}{i+1} m \quad(i=1, \ldots, N-1), \quad \text { and } \quad \mu_{N}=N m .
$$

The product of the Gaussian single-particle wave packets can then be written as a product of Gaussians depending on the relative and center-of-mass coordinates:

$$
\prod_{i=1}^{N} \varphi_{\mathbf{s}_{i}}^{\nu}\left(\mathbf{r}_{i}\right)=\prod_{i=1}^{N} \varphi_{\mathbf{S}_{i}}^{\gamma_{i}}\left(\mathbf{x}_{i}\right)
$$

with

$$
\gamma_{i}=\frac{\mu_{i} \omega}{2 \hbar} .
$$

By using Eq. (31) and noting that the last factor of the product depends only on the centerof-mass coordinate, which is symmetric under the exchange of nucleons, the $N$-nucleon wave function can be rewritten as

$$
\Phi_{S M_{S} T M_{T}}\left(\mathbf{s}_{1}, \ldots, \mathbf{s}_{N}\right)=\Psi_{S M_{S} T M_{T}}\left(\mathbf{S}_{1}, \ldots, \mathbf{S}_{N-1}\right) \varphi_{\mathbf{S}_{N}}^{\gamma_{N}}\left(\mathbf{x}_{N}\right),
$$

which defines the intrinsic function that depends solely on the relative coordinates,

$$
\Psi_{S M_{S} T M_{T}}\left(\mathbf{S}_{1}, \ldots, \mathbf{S}_{N-1}\right)=\mathcal{A}\left\{\varphi_{\mathbf{S}_{1}}^{\gamma_{1}}\left(\mathbf{x}_{1}\right), \ldots, \varphi_{\mathbf{S}_{N-1}}^{\gamma_{N-1}}\left(\mathbf{x}_{N-1}\right) \chi_{S M_{S}} \mathcal{X}_{T M_{T}}\right\}
$$

As will be shown in the next subsection, this function serves as a generating function of the correlated Gaussian basis.

The Hamiltonian of Eq. (21) can be recast to the relative plus center-of-mass terms as

$$
H=\left(\sum_{i=1}^{N-1} \frac{\mathbf{P}_{i}^{2}}{2 \mu_{i}}+\sum_{i<j}^{N} V_{i j}\right)+\frac{\mathbf{P}_{N}^{2}}{2 \mu_{N}} \equiv H_{r e l}+T_{c m},
$$

where $\mathbf{P}_{i}$ is the momentum canonically conjugate to the Jacobi coordinate $\mathbf{x}_{i}$. The matrix elements of the Hamiltonian are then 


$$
\begin{aligned}
& \left\langle\Phi_{S M_{S} T M_{T}}\left(\mathbf{s}_{1}, \ldots, \mathbf{s}_{N}\right)|H| \Phi_{S^{\prime} M_{S}^{\prime} T^{\prime} M_{T}^{\prime}}\left(\mathbf{s}_{1}^{\prime}, \ldots, \mathbf{s}_{N}^{\prime}\right)\right\rangle \\
& =\left\langle\Psi_{S M_{S} T M_{T}}\left(\mathbf{S}_{1}, \ldots, \mathbf{S}_{N-1}\right)\left|H_{r e l}\right| \Psi_{S^{\prime} M_{S}^{\prime} T^{\prime} M_{T}^{\prime}}\left(\mathbf{S}^{\prime}{ }_{1}, \ldots, \mathbf{S}^{\prime}{ }_{N-1}\right)\right\rangle\left\langle\varphi_{\mathbf{S}_{N}}^{\gamma_{N}} \mid \varphi_{\mathbf{S}^{\prime} N}^{\gamma_{N}}\right\rangle \\
& +\left\langle\Psi_{S M_{S} T M_{T}}\left(\mathbf{S}_{1}, \ldots, \mathbf{S}_{N-1}\right) \mid \Psi_{S^{\prime} M_{S}^{\prime} T^{\prime} M_{T}^{\prime}}\left(\mathbf{S}^{\prime}{ }_{1}, \ldots, \mathbf{S}_{N-1}^{\prime}\right)\right\rangle\left\langle\varphi_{\mathbf{S}_{N}}^{\gamma_{N}}\left|T_{c m}\right| \varphi_{\mathbf{S}^{\prime}{ }_{N}}^{\gamma_{N}}\right\rangle .
\end{aligned}
$$

The matrix elements of the Hamiltonian $H_{r e l}$ can be expressed by integrating over $\mathbf{S}_{N}$

$$
\begin{aligned}
& \left\langle\Psi_{S M_{S} T M_{T}}\left(\mathbf{S}_{1}, \ldots, \mathbf{S}_{N-1}\right)\left|H_{r e l}\right| \Psi_{S^{\prime} M_{S}^{\prime} T^{\prime} M_{T}^{\prime}}\left(\mathbf{S}_{1}^{\prime}, \ldots, \mathbf{S}_{N-1}^{\prime}\right)\right\rangle \\
& =\left(\frac{\gamma_{N}}{2 \pi}\right)^{3 / 2} \int d \mathbf{S}_{N}\left\langle\Phi_{S M_{S} T M_{T}}\left(\mathbf{s}_{1}, \ldots, \mathbf{s}_{N}\right)|H| \Phi_{S^{\prime} M_{S}^{\prime} T^{\prime} M_{T}^{\prime}}\left(\mathbf{s}_{1}^{\prime}, \ldots, \mathbf{s}_{N}^{\prime}\right)\right\rangle,
\end{aligned}
$$

where use has been made of the single-particle matrix elements in Appendix A and the formula

$$
\int d \mathbf{S}_{N}\left(-\frac{\gamma_{N}}{2}\left(\mathbf{S}_{N}-\mathbf{S}_{N}^{\prime}\right)^{2}\right)^{n} \mathrm{e}^{-\frac{\gamma_{N}}{2}\left(\mathbf{S}_{N}-\mathbf{S}_{N}^{\prime}\right)^{2}}=\left(-\frac{1}{2}\right)^{n}(2 n+1) ! !\left(\frac{2 \pi}{\gamma_{N}}\right)^{\frac{3}{2}} .
$$

Equation (37) shows that the matrix elements between the intrinsic function can be obtained in a simple way by factorizing the $\mathbf{S}_{N}$-dependent terms from the matrix elements in the single-particle basis: The quadratic forms in Eqs. (22), (24) and (25), with the help of Eq. (36), should take the form

$$
\tilde{\mathbf{s}} A_{i}^{(k)} \mathbf{s}=\tilde{\mathbf{S}} B_{i}^{(k)} \mathbf{S}+\gamma_{N}\left(\mathbf{S}_{N}-\mathbf{S}_{N}^{\prime}\right)^{2} \quad(k=o, p) .
$$

Here the $(2 N-2)$-element column vector $\mathbf{S}$ is an abbreviation for the set of the Jacobi generator-coordinate vectors $\left(\mathbf{S}_{1}, \ldots, \mathbf{S}_{N-1}, \mathbf{S}^{\prime}{ }_{1}, \ldots, \mathbf{S}_{N-1}^{\prime}\right)$. The matrix $B_{i}^{(k)}$ is the $(2 N-2) \times$ $(2 N-2)$ symmetric matrix defined by dropping the $N$ th and $2 N$ th rows and columns of the matrix $\tilde{T} A_{i}^{(k)} T$, where

$$
T=\left(\begin{array}{cc}
U^{-1} & 0 \\
0 & U^{-1}
\end{array}\right)
$$

It is clear from Eq. (36) that the polynomials $P_{i}(\mathbf{s}, \mathbf{r})$ and the vectors $\mathbf{d}_{i}$ defined in Eq. (25) can depend only on the relative generator coordinates. The dependence of the matrix elements on the center-of-mass variables, $\mathbf{S}_{N}$ and $\mathbf{S}_{N}^{\prime}$, can, therefore, be factorized and the integration in Eq. (37) reduces to such a simple form as the one in Eq. (38). After the integration over $\mathbf{S}_{N}$ the matrix elements can be expressed in terms of the Jacobi generator coordinates in the form:

$$
\begin{gathered}
\left\langle\Psi_{S M_{S} T M_{T}}\left(\mathbf{S}_{1}, \ldots, \mathbf{S}_{N-1}\right) \mid \Psi_{S M_{S} T M_{T}}\left(\mathbf{S}_{1}^{\prime}, \ldots, \mathbf{S}^{\prime}{ }_{N-1}\right)\right\rangle=\sum_{i=1}^{n_{o}} C_{i}^{(o)} \mathrm{e}^{-\frac{1}{2} \tilde{\mathbf{S}} B_{i}^{(o)} \mathbf{S}} \\
\left\langle\Psi_{S M_{S} T M_{T}}\left(\mathbf{S}_{1}, \ldots, \mathbf{S}_{N-1}\right)\left|\sum_{i=1}^{N} \frac{\mathbf{P}_{i}^{2}}{2 \mu_{i}}\right| \Psi_{S M_{S} T M_{T}}\left(\mathbf{S}^{\prime}, \ldots, \mathbf{S}^{\prime}{ }_{N-1}\right)\right\rangle \\
=\frac{\hbar \omega}{2} \sum_{i}^{n_{o}} C_{i}^{(o)}\left(\frac{3}{2}(N-1)-\frac{1}{2} \tilde{\mathbf{S}} B_{i}^{(o)} \mathbf{S}\right) \mathrm{e}^{-\frac{1}{2} \tilde{\mathbf{S}} B_{i}^{(o)} \mathbf{S}}
\end{gathered}
$$




$$
\begin{aligned}
& \left\langle\Psi_{S M_{S} T M_{T}}\left(\mathbf{S}_{1}, \ldots, \mathbf{S}_{N-1}\right)\left|\sum_{i<j}^{N} V_{i j}\right| \Psi_{S^{\prime} M_{S}^{\prime} T^{\prime} M_{T}^{\prime}}\left(\mathbf{S}_{1}^{\prime}, \ldots, \mathbf{S}_{N-1}^{\prime}\right)\right\rangle \\
& =\int d \mathbf{r} V(r) \mathrm{e}^{-\nu r^{2}} \sum_{i=1}^{n_{p}} C_{i}^{(p)} P_{i}(\mathbf{S}, \mathbf{r}) \mathrm{e}^{-\frac{1}{2} \tilde{\mathbf{S}} B_{i}^{(p)} \mathbf{S}+\mathbf{D}_{i} \cdot \mathbf{r}} .
\end{aligned}
$$

Here the convention of renumbering the set of the vectors $\left(\mathbf{S}_{1}^{\prime}, \ldots, \mathbf{S}_{N-1}^{\prime}\right)$ of the ket as $\left(\mathbf{S}_{N}, \ldots, \mathbf{S}_{2 N-2}\right)$ is used to simplify the notation and thus $\mathbf{S}$ stands for the set of the vectors $\left(\mathbf{S}_{1}, \ldots, \mathbf{S}_{2 N-2}\right)$. The vector $\mathbf{D}_{i}$ is given by $\sum_{j=1}^{2 N-2} \hat{D}_{(i) j} \mathbf{S}_{j}$, where the $\hat{D}_{(i) j}$ 's are formed from the elements of the row vector $\tilde{\mathcal{D}}_{(i)} T$ by omitting its $N$ th and $2 N$ th columns. The column vector $\mathcal{D}_{(i)}$ is defined in Eq. (26).

\section{Integral transformation to the correlated Gaussian basis}

Here we show how to evaluate the matrix elements in the correlated Gaussian basis. Let us choose the correlated Gaussians with the form of Eq. (6) and introduce the function

$$
f_{K L M}(\mathbf{u}, \mathbf{x}, A)=\eta_{K L M}(\mathbf{u}, \mathbf{x}) \mathrm{e}^{-\frac{1}{2} \tilde{\mathbf{x}} A \mathbf{x}},
$$

where

$$
\eta_{K L M}(\mathbf{u}, \mathbf{x})=v^{2 K+L} Y_{L M}(\hat{\mathbf{v}}), \quad \text { with } \quad \mathbf{v}=\sum_{i=1}^{N-1} u_{i} \mathbf{x}_{i}=\tilde{\mathbf{u}} \mathbf{x}
$$

and where $\tilde{\mathbf{u}}=\left(u_{1}, \ldots, u_{N-1}\right)$. Note that $\mathbf{u}$ is a set of $(N-1)$ real numbers, whereas $\mathbf{x}$ are the $(N-1)$ Jacobi coordinates. The calculation of the matrix elements becomes simple if one uses a generating function of the correlated Gaussian. In fact, the following function $g$ is found to be most convenient to generate the function $f$ :

$$
f_{K L M}(\mathbf{u}, \mathbf{x}, A)=\frac{1}{B_{K L}} \int d \hat{\mathbf{t}} Y_{L M}(\hat{\mathbf{t}})\left(\frac{d^{2 K+L}}{d \alpha^{2 K+L}} g(\alpha, \mathbf{t} ; \mathbf{u}, \mathbf{x}, A)\right)_{\substack{\alpha=0 \\ t=|\mathbf{t}|=1}}
$$

where

$$
\begin{gathered}
g(\alpha, \mathbf{t} ; \mathbf{u}, \mathbf{x}, A)=\mathrm{e}^{-\frac{1}{2} \tilde{\mathbf{x}} A \mathbf{x}+\alpha \mathbf{v} \cdot \mathbf{t}}, \\
B_{n l}=\frac{4 \pi(2 n+l) !}{2^{n} n !(2 n+2 l+1) ! !} .
\end{gathered}
$$

Equation (46) is easily proved by using the simple formula

$$
(\mathbf{v} \cdot \mathbf{t})^{k}=v^{k} t^{k} \sum_{\substack{n, l \geq 0 \\ 2 n+l=k}} B_{n l} \sum_{m=-l}^{l} Y_{l m}(\hat{\mathbf{v}}) Y_{l m}(\hat{\mathbf{t}})^{*} .
$$

For a case where the function $\theta_{L M_{L}}(\mathbf{x})$ of Eq. (7) is needed, the generating function $g$ of Eq. (47) must be generalized to include another factor $\alpha^{\prime} \mathbf{v}^{\prime} \cdot \mathbf{t}^{\prime}$. Since the following derivation remains essentially unaffected by this generalization, we will assume Eq. (6) as $\theta_{L M_{L}}(\mathbf{x})$. 
The generating function $g$ can be related to the product of the Gaussians centered around $\left\{\mathbf{S}_{i} ; i=1, \ldots, N-1\right\}$ through an integral transformation. To show this, we express the product of the Gaussian wave packets as

$$
\prod_{i=1}^{N-1} \varphi_{\mathbf{S}_{i}}^{\gamma_{i}}\left(\mathbf{x}_{i}\right)=\left(\frac{\operatorname{det} \Gamma}{\pi^{N-1}}\right)^{3 / 4} \mathrm{e}^{-\frac{1}{2} \tilde{\mathbf{x}} \Gamma \mathbf{x}+\tilde{\mathbf{x}} \Gamma \mathbf{S}_{H}-\frac{1}{2} \tilde{\mathbf{S}}_{H} \Gamma \mathbf{S}_{H}}
$$

with an $(N-1) \times(N-1)$ diagonal matrix

$$
\Gamma=\left(\begin{array}{cccc}
2 \gamma_{1} & 0 & \ldots & 0 \\
0 & 2 \gamma_{2} & & \vdots \\
\vdots & & & \vdots \\
0 & \ldots & \ldots & 2 \gamma_{N-1}
\end{array}\right)
$$

where $\mathbf{S}_{H}$ stands for the set of the generator coordinate vectors $\left(\mathbf{S}_{1}, \ldots, \mathbf{S}_{N-1}\right)$. By using the familiar formula of the $n$-dimensional Gaussian integration

$$
\int d \mathbf{x} \mathrm{e}^{-\frac{1}{2} \tilde{\mathbf{x}} A \mathbf{x}+\tilde{\mathbf{T}} \mathbf{x}}=\left(\frac{(2 \pi)^{n}}{\operatorname{det} A}\right)^{3 / 2} \mathrm{e}^{\frac{1}{2} \tilde{\mathbf{T}} A^{-1} \mathbf{T}}
$$

it is easy to prove the following equation by a direct calculation

$$
g(\alpha, \mathbf{t} ; \mathbf{u}, \mathbf{x}, A)=\left(\frac{(\operatorname{det} \Gamma)^{3 / 2}}{(4 \pi)^{(N-1) / 2} \operatorname{det}(\Gamma-A)}\right)^{3 / 2} \mathrm{e}^{-\frac{1}{2} \tilde{\mathbf{T}} C \mathbf{T}} \int d \mathbf{S}_{H} \mathrm{e}^{-\frac{1}{2} \tilde{\mathbf{S}}_{H} Q \mathbf{S}_{H}+\tilde{\mathbf{T}} \mathbf{S}_{H}}\left(\prod_{i=1}^{N-1} \varphi_{\mathbf{S}_{i}}^{\gamma_{i}}\left(\mathbf{x}_{i}\right)\right)
$$

where $\tilde{\mathbf{T}}=\left(\mathbf{T}_{1}, \ldots, \mathbf{T}_{N-1}\right)$, and

$$
\begin{gathered}
C=\Gamma^{-1}(\Gamma-A) \Gamma^{-1}, \quad Q=C^{-1}-\Gamma, \\
\mathbf{T}_{i}=\alpha \mathbf{t} \sum_{j=1}^{N-1}(\Gamma C)_{i j}^{-1} u_{j} \quad(i=1, \ldots, N-1) .
\end{gathered}
$$

By combining Eqs. (46) and (53), the correlated Gaussian basis is found to be generated from the intrinsic state given in Eq. (34) by the integral transformation

$$
\begin{aligned}
& \mathcal{A}\left\{f_{K L M}(\mathbf{u}, \mathbf{x}, A) \chi_{S M_{S}} \mathcal{X}_{T M_{T}}\right\}=\frac{1}{B_{K L}}\left(\frac{(\operatorname{det} \Gamma)^{3 / 2}}{(4 \pi)^{(N-1) / 2} \operatorname{det}(\Gamma-A)}\right)^{3 / 2} \\
\times & \int d \hat{\mathbf{t}} Y_{L M}(\hat{\mathbf{t}})\left(\frac{d^{2 K+L}}{d \alpha^{2 K+L}} \mathrm{e}^{-\frac{1}{2} \tilde{\mathbf{T}} C \mathbf{T}} \int d \mathbf{S}_{H} \mathrm{e}^{-\frac{1}{2} \tilde{\mathbf{S}}_{H} Q \mathbf{S}_{H}+\tilde{\mathbf{T}} \mathbf{S}_{H}} \Psi_{S M_{S} T M_{T}}\left(\mathbf{S}_{1}, \ldots, \mathbf{S}_{N-1}\right)\right)_{\substack{\alpha=0 \\
t=1}}
\end{aligned}
$$

The matrix elements between the correlated Gaussians are now easily obtained by the integral transformation from those expressed in terms of the relative generator coordinates 
S. Using Eq. (56) gives a general formula to calculate a matrix element for any translationinvariant operator $\mathcal{O}$

$$
\begin{aligned}
& \left\langle\mathcal{A}\left\{f_{K L M}(\mathbf{u}, \mathbf{x}, A) \chi_{S M_{S}} \mathcal{X}_{T M_{T}}\right\}|\mathcal{O}| \mathcal{A}^{\prime}\left\{f_{K^{\prime} L^{\prime} M^{\prime}}\left(\mathbf{u}^{\prime}, \mathbf{x}, A^{\prime}\right) \chi_{S^{\prime} M_{S}^{\prime}} \mathcal{X}_{T^{\prime} M_{T}^{\prime}}\right\}\right\rangle \\
= & \frac{1}{B_{K L} B_{K^{\prime} L^{\prime}}^{\prime}}\left(\frac{(\operatorname{det} \Gamma)^{3}}{(4 \pi)^{(N-1)} \operatorname{det}(\Gamma-A) \operatorname{det}\left(\Gamma-A^{\prime}\right)}\right)^{3 / 2} \\
\times & \iint d \hat{\mathbf{t}} d \hat{\mathbf{t}}^{\prime} Y_{L M}(\hat{\mathbf{t}})^{*} Y_{L^{\prime} M^{\prime}}\left(\hat{\mathbf{t}}^{\prime}\right)\left(\frac{d^{2 K+L+2 K^{\prime}+L^{\prime}}}{d \alpha^{2 K+L} d \alpha^{\prime 2 K^{\prime}+L^{\prime}}} \mathrm{e}^{-\frac{1}{2} \tilde{\mathbf{T}} C \mathbf{T}}\right. \\
\times & \left.\int d \mathbf{S} \mathrm{e}^{-\frac{1}{2} \tilde{\mathbf{S}} Q \mathbf{S}+\tilde{\mathbf{T}} \mathbf{S}}\left\langle\Psi_{S M_{S} T M_{T}}\left(\mathbf{S}_{1}, \ldots, \mathbf{S}_{N-1}\right)|\mathcal{O}| \Psi_{S^{\prime} M_{S}^{\prime} T^{\prime} M_{T}^{\prime}}\left(\mathbf{S}_{1}^{\prime}, \ldots, \mathbf{S}_{N-1}^{\prime}\right)\right\rangle\right)_{\substack{\alpha=\alpha^{\prime}=0 \\
t=t^{\prime}=1}},
\end{aligned}
$$

where $\tilde{\mathbf{S}}=\left(\mathbf{S}_{1}, \ldots, \mathbf{S}_{N-1}, \mathbf{S}_{1}^{\prime}=\mathbf{S}_{N}, \ldots, \mathbf{S}_{N-1}^{\prime}=\mathbf{S}_{2 N-2}\right)$ and the matrices, $C$ and $Q$, and the vectors $\mathbf{T}$ in Eq. (57), although the same notation is used as in Eqs. (54) and (55), are extended to include the corresponding primed quantities of the ket, that is,

$$
C \longrightarrow\left(\begin{array}{cc}
C & 0 \\
0 & C^{\prime}
\end{array}\right), \quad Q \longrightarrow\left(\begin{array}{cc}
Q & 0 \\
0 & Q^{\prime}
\end{array}\right), \quad \mathbf{T} \longrightarrow\left(\begin{array}{c}
\mathbf{T} \\
\mathbf{T}^{\prime}
\end{array}\right)
$$

As is shown in Eqs. (41)-(43), the $\mathbf{S}$-dependence of the matrix elements is rather simple and the integration over $\mathbf{S}$ is done analytically. Since the variables $\alpha, \alpha^{\prime}, \mathbf{t}$, and $\mathbf{t}^{\prime}$ appear

only through the vector $\mathbf{T}$, those operations with respect to them as implied in Eq. (57) are performed systematically. An illustrative example is given in Appendix D. The coupling of the orbital and spin angular momenta causes no difficulty. It is very satisfactory aesthetically that matrix elements between the basis functions with any sets of the relative coordinates can be evaluated in a unified framework without any extra transformation of the coordinates. The choice of the set of the relative coordinates amounts to the choice of the matrix $A$.

\section{Extension to the system of particles of unequal masses}

In this subsection we remark the modifications needed to treat few-particle systems containing particles of unequal masses. As you will see, most of the formulation presented in subsections III.A-III.C remains unchanged. Suppose that the masses of the $N$ particles are $m_{1}, m_{2}, \ldots, m_{N}$. The width parameter of the Gaussian wave packet is to be changed to

$$
\nu_{i}=\frac{m_{i} \omega}{2 \hbar} \quad(i=1, \ldots, N) .
$$

The overlap and Hamiltonian matrix elements are obtained as a function of the generator coordinates, $\left(\mathbf{s}_{1}, \ldots, \mathbf{s}_{N}, \mathbf{s}_{1}^{\prime}, \ldots, \mathbf{s}_{N}^{\prime}\right)$, in a form similar to the previous case. In this general case of unequal masses, the matrix $U$ in Eq. (28) which defines a set of the Jacobi coordinates must be generalized to

$$
U=\left(\begin{array}{ccccc}
-1 & 1 & 0 & \ldots & 0 \\
-\frac{m_{1}}{m_{12}} & -\frac{m_{2}}{m_{12}} & 1 & \ldots & 0 \\
\vdots & & & & \vdots \\
-\frac{m_{1}}{m_{12 \cdots N-1}} & -\frac{m_{2}}{m_{12 \cdots N-1}} & \cdots & \cdots & 1 \\
\frac{m_{1}}{m_{12 \cdots N}} & \frac{m_{2}}{m_{12 \cdots N}} & \cdots & \cdots & \frac{m_{N}}{m_{12 \cdots N}}
\end{array}\right),
$$


where $m_{12 \cdots i}=m_{1}+m_{2}+\cdots+m_{i}$. The reduced masses corresponding to this $U$ are accordingly given, instead of Eq. (30), by

$$
\mu_{i}=\frac{m_{i+1} m_{12 \cdots i}}{m_{12 \cdots i+1}} \quad(i=1, \ldots, N-1), \quad \text { and } \quad \mu_{N}=m_{12 \cdots N}
$$

What is important is to realize that Eq. (31), most crucial in eliminating the center-of-mass motion, still holds even with these modifications as

$$
\prod_{i=1}^{N} \varphi_{\mathbf{s}_{i}}^{\nu_{i}}\left(\mathbf{r}_{i}\right)=\prod_{i=1}^{N} \varphi_{\mathbf{S}_{i}}^{\gamma_{i}}\left(\mathbf{x}_{i}\right) .
$$

It is then easy to see that the rest of all the formulae are exactly the same as the case of equal masses. We can conclude that the needed modifications noted above are rather trivial and simple but still assures the elimination of the center-of-mass motion. As a simple example of unequal masses, the system of $t+d+\mu^{-}-$molecule will be considered in subsection IV.B.

\section{NUMERICAL RESULTS}

This section is devoted to present the solutions of various $N=2-7$-body problems by applying the method described above. To test the method, different potentials (Yukawa, Gauss and Coulomb) have been used for bosonic and fermionic systems. Some of the examples shown here has its own physical significance, and some other solutions may be considered as benchmark test and might be useful in comparison of various few-body methods. One can expect that, besides the VMC [7,8] and GFMC [9,10], other methods will also be extended to treat more than $N=4$-particle systems. As only a few solutions are at present available for simple potentials, the examples listed here may help to test other methods.

As was discussed in subsection II.A, there is no restriction on the choice of the orthogonal matrix $G$. We have found, however, that those special rotation matrices which connect different sets of the relative coordinates especially suitable (see also [17]) and will use them in what follows. This greatly helps to reduce the number of parameters of $G$. In the stochastic selection of the basis elements, these special matrices $G$ and the parameters of the diagonal matrix $A^{\prime}$ are randomly chosen. The vector $\mathbf{u}$ in Eq. (6) is also a variational parameter. To avoid an excessively large number of variational parameters we limited uvector values to those which are needed to generate the function $\theta_{L M_{L}}(\mathbf{x})$ of Eq. (5) for a given set of angular momenta. Comparison of our calculation with others confirms that these limitations have not deteriorated the accuracy of the present calculation, that is, our trial function is flexible enough. In the calculation the sets of angular momenta (i.e., the sets of these special $\mathbf{u}$ vectors) are also randomly chosen. The main advantage of using $\mathbf{u}$ lies in the simple and systematic evaluation of the matrix elements from the point of view of both analytical and numerical calculations. Further test calculations will be needed to explore the utility of $\mathbf{u}$ as a variational parameter.

Because the dependence of the matrix elements on the variational parameters is known as is shown in Appendix D, one can organize the numerical calculation involved in the random search economically. A change of the values of the parameters does not require a recalculation 
of the whole matrix element. Once they have been calculated for one set of values, to calculate them for many more requires virtually no time. The average computational time is 10 minutes for a four-body and 2 hours for a six-body calculation on the VPP500 computer of RIKEN.

\section{A. Few-nucleon systems}

We have performed model calculations adopting different central potentials as nucleonnucleon interaction. Some of these model problems have already been solved to high accuracy by various methods and therefore we can directly compare the solutions. The potentials used for comparison of different methods are (i) the Malfliet-Tjon (MT-V) potential [29], which has been most extensively used as benchmark test in few-body calculations, (ii) the Volkov "super-soft" core potential [30, (iii) the Afnan-Tang S3 (ATS3) potential [31] which exhibits a strong repulsive core and incorporates a difference between the spin-singlet and spin-triplet channels, (iv) and the Minnesota potential [32] which reproduces the most important low-energy nucleon-nucleon phase shifts. The Volkov and MT-V potentials are spin-independent, while the ATS3 and Minnesota potentials are spin-dependent. The parameters of the interactions are tabulated in Table I. We choose $\hbar^{2} / m=41.47 \mathrm{MeV} \mathrm{fm}{ }^{2}$. The Coulomb interaction is included only in calculations with the Minnesota potential where point charges are assumed and $e^{2}=1.44 \mathrm{MeV}$ fm.

The spins (and isospins) of the nucleons are coupled through successive intermediate couplings. The spin couplings up to $N=7$ nucleons are tabulated in Table II. One naturally expects and test calculations show that, without spin-isospin coupling, the energy convergence is much slower. The number of spin-isospin configurations rapidly increases with $N$. In the case of ${ }^{6} \mathrm{He}$, for example, assuming $S=0$ and $T=1$, the wave function has $5 \times 9=45$ spin-isospin components. The number of components becomes even higher if the interaction has non-central spin-orbit, tensor, etc. parts. The nonlinear parameters are not optimized with respect to spin-isospin components, but rather, for each trial choice of the matrix $A$, we select the spin-isospin component that gives the lowest energy. Because the matrix elements between different spin-isospin components differ only in linear factors $\left(c_{\kappa}\right.$ in Eq. (20)), the calculation of the matrix elements of each spin-isospin component of the wave function requires essentially the same computational effort as that needed by the calculation for only one component.

Each calculation has been repeated several times starting from different random points to check the energy convergence. The energy as a function of the number of basis states is shown in Fig. 2 for the case of ${ }^{6} \mathrm{Li}$ with the Volkov potential. The energies on different random paths, after a few initial steps, approach to each other and converge to the final solution. The energy difference between two random paths as well as the tangent of the curves give us some information on the accuracy of the method on a given size of the basis. The root mean square (rms) radius of the few-nucleon system is calculated in each step and found to be rapidly convergent to its final value. By increasing the basis size the results can be arbitrarily improved when needed.

The number of basis states required to reach energy convergence increases with the number of particles but it depends on the form of the interaction as well. This latter 
property is illustrated in Fig. 3 for the case of the $\alpha$-particle. The soft-core Volkov potential shows rapid convergence, while the hard-core ATS3 interaction requires more basis states to get an accurate solution. The relatively fast convergence for the MT-V potential of a strong repulsion can be explained by the simplicity - the spin-independent nature - of this interaction.

In the following we show tables for the ground-state energies $E$ and point matter rms radii $\left\langle r^{2}\right\rangle^{1 / 2}$. The basis dimension $\mathcal{K}$ of the SVM listed in the tables is such that, beyond it, the energy and the radius do not change in the digits shown. Table III shows our results (SVM), together with results of others, for the application of the spin-averaged MT-V potential [29] to $N=2-7$-nucleon systems. For three-body systems, the solution of the Faddeev equation is known to be the method of choice, but the SVM can easily yield the same accurate energy. As the MT-V potential is a preferred benchmark test of the few-body calculations, there are numerous solutions available. Table III includes a few of the most accurate results. The nice agreement for four-nucleon case corroborates that the SVM is as accurate as the direct solution of the FY equations [33], the method of the Amalgamation of Two-body correlations into Multiple Scattering (ATMS) [4] process or the VMC [34] and GFMC [9] method. The basis used in the Coupled-Rearrangement-Channel Gaussian-basis (CRCG) variational method [6] is similar to that of the SVM, but the Gaussian parameters follow geometric progressions. The fact that the basis size needed in the SVM is much smaller proves the efficiency of the selection procedure. The results of the VMC calculation for the five- and six-nucleon systems are also in good agreement with the results of SVM. The MT$\mathrm{V}$ potential has no exchange term; therefore, unlike the nature, it renders the five-nucleon system bound, and the nucleus tends to collapse as the binding energy increases with the number of particles.

The next example is the Volkov potential which, due to its very soft core, is the most readily solvable case. This simple potential is widely used in model calculations for light nuclei. As one sees in Table IV, the results of SVM agree with those of other calculations, especially with the one using hyperspherical harmonics $(\mathrm{HH})$ functions. The number of basis states needed to reach convergence is remarkably smaller than in the case of the MT-V potential. Without the Majorana exchange term $(M=0)$ this potential also leads to a collapsing system. By setting the Majorana parameter to $M=0.6$, a commonly used value to get the correct binding energy, one may obtain more reasonable energies. The Volkov potential with $M=0.6$ does not change the energies of $N=2-4$ nuclei, does not bind ${ }^{5} \mathrm{Li}$ in accordance with the nature, but does bind the ${ }^{6} \mathrm{Li}$ ground state $\left(E=-31.82 \mathrm{MeV},\left\langle r^{2}\right\rangle^{1 / 2}\right.$ $=2.69 \mathrm{fm})$.

Another potential that is often used in test calculation is the ATS3 potential. We have challenged the SVM to get solution for this case because, unlike the Volkov, this spindependent potential has a relatively strong repulsive core (see Table I). The solution, although on a somewhat larger basis, can easily be obtained, and it is in good agreement with those of other methods in the $N=3$ system as shown in Table V. We note, however, that the energy of the SVM is significantly lower than the ones of other methods for the $\alpha$-particle. This may be due to the strong repulsion of this potential. For example, the FY calculation [33] agrees with ours for the MT-V potential, but shows a noticeable difference in the case of the ATS3 potential. The variational calculation [16] using a correlation factor 
also misses considerable energy for the ATS3 potential. Surprisingly, inspite of its exchange part, this potential also binds the five-nucleon system and overbinds the six-nucleon systems very much.

The last example for the few-nucleon system uses the more realistic Minnesota potential [32], which is a central interaction of Gaussian form, containing space-, spin-, and isospin-exchange operators (see Table I). The Minnesota potential has often been used in cluster-model calculations of light nuclei. Table VI shows results with this potential, where the Coulomb interaction between protons is also included. All possible spin and isospin configurations are allowed for and all spherical harmonics that give non-negligible contribution are included in $\theta_{L M_{L}}(\mathbf{x})$. Since the method has proved to be accurate and reliable for other potentials, it is justifiable to view these results as testing the interaction rather than the method, and hence the results are compared with experimental data. The energy and the radius of triton and $\alpha$-particle converge, with small bases, to realistic values. The Minnesota potential, correctly, does not bind the $N=5$ system, but it binds ${ }^{6} \mathrm{He}$ and slightly overbinds ${ }^{6} \mathrm{Li}$. The radius of ${ }^{6} \mathrm{He}$ is found to be much larger than that of ${ }^{4} \mathrm{He}$, consistently with the halo structure of ${ }^{6} \mathrm{He}$ [17]. It is for the first time that the Minnesota force is tested without assuming any cluster structure or restricting the model space by any other bias. The agreement is surprisingly good not only with experiment but also with cluster-model calculations for all nuclei [38].

It is interesting to note that none of these simple potentials binds the four-neutron system. The Volkov potential, for example, is so strong that it binds the singlet two-neutron system, but it does not allow the neutrons to form a four-neutron bound state due to the Pauli principle.

As an example for bosonic nuclear few-body system, we consider the case of structureless $\alpha$-particles interacting via the state-independent potential;

$$
V(r)=500 \exp \left[-(0.7 r)^{2}\right]-130 \exp \left[-(0.475 r)^{2}\right] \quad(\mathrm{MeV}),
$$

where $r$ is in fm. This potential is taken from Ali-Bodmer's $S$-state potential [39]. It has a repulsive core which is about $370 \mathrm{MeV}$ high and extends up to $2 \mathrm{fm}$. The repulsive core prevents the $\alpha$-particles from collapsing. The results are compared in Table VII. Our calculation agrees with the ATMS result for the $N=3$ and 4 systems.

\section{B. Coulombic systems}

The results for the long range $1 / r$ potential are collected in this subsection. The first example is the polyelectric system $\left(m e^{+}, n e^{-}\right)$. The possibility that $m$ positrons and $n$ electrons form a bound system was originally suggested by Wheeler [40] and this question has been extensively studied since then. Besides the trivial and analytically solvable $m=1$ and $n=1$ case, the existence of the positronium negative ion $(m=1, n=2)$ was also predicted by Wheeler [40]. Dozens of works have attempted to solve the $e^{+}, e^{-}, e^{-}$Coulombic three-

body problem, continuously refining the accuracy of the calculated binding energy 41, 42. Despite of numerous attempts, no one has obtained bound states for the polyelectric system of more than four particles. The positronium negative ion has experimentally been observed 43. 
The binding energy of the positronium molecule $\left(2 e^{+}, 2 e^{-}\right)$was first calculated by Hylleraas and Ore [44]. To date, the positronium molecule has not been directly observed, and this fact intensifies the theoretical interest to solve this Coulombic four-body problem. This molecule is short-lived because the electron and positron may annihilate. Unlike the positronium ion, the positronium molecule is neutral, and therefore the best chance to distinguish it from the positronium itself is related to their different lifetimes. The QED formulae to determine the probability of a pair annihilation in the positronium molecule through a $k$-photon process $(k=0,1,2, \ldots)$ would require a highly accurate wave function [45].

In Table VIII we compare our results to the most precise calculations found in the literature. The correlated Gaussian function without the polynomial part $(K=0$ in Eq. (6)) is known to poorly represent the Coulomb cusps [18]. To improve the cusp properties the trial function with $K=0,1,2$ polynomials has been used.

As is shown in Table VIII, our calculation reproduces the first six digits of the variational calculation of Ref. [41] for the ground state of $\left(2 \mathrm{e}^{+}, \mathrm{e}^{-}\right)$, and the rms radius also agrees with it. There are two recent variational calculations [19,46] for the positronium molecule using the correlated Gaussian functions. In these works the nonlinear parameters were determined by optimatization. To compare our calculations to theirs directly, the value of $K=0$ was chosen and the same basis size $(\mathcal{K}=300)$ was used. Our result is slightly better than the energy obtained by them and this reinforces the reliability and powerfulness of the random selection of the nonlinear parameters. The number of nonlinear parameters of this case is $\mathcal{K} \times(4 \times 3) / 2=1800$. The complete optimatization of the parameters is, of course, superior to the SVM. Test calculations show that, provided the number of parameters is low, that is, a full optimatization is feasible, the optimatization finds lower energy. But when the number of parameters becomes high, the full optimatization becomes less and less practical partly because it fails to find true minimum and partly because the computation becomes too excessive.

We found no bound states for the $\left(3 e^{+}, 2 e^{-}\right)$and $\left(3 e^{+}, 3 e^{-}\right)$systems. The energy of $\left(3 e^{+}, 3 e^{-}\right)$, for example, converges to the sum of the energy of a dipositronium molecule and of a positronium (0.515989 a.u. +0.25 a.u. $=0.765989$ a.u.). Allowing the selection of the parameters from a larger region increases the rms radius, which is typical of an unbound state. The system of a negative and a positive positronium ion thus forms no bound state but dissociates into a dipositronium molecule and a positronium.

Calculations for $L=1$ state also fails to find a bound system. This result entails that the Coulomb force cannot bind more than four particles out of identical charged fermions and their antiparticles.

To examine the role of the Pauli principle in preventing five-electron-positron system from forming bound states, we repeated the same calculation replacing the fermions by bosonic equivalents. On a different scale, these systems may be identified, e.g., as the systems of $\pi^{-}$ and $\pi^{+}$with their strong interaction neglected [47]. Such bosons turn out to form bound states even for $N=5$. As is expected, the radius of the charged boson system decreases as the number of particles increases. It is interesting to note that the energies of bosonic and fermionic systems are equal for $N=3$ and for $N=4$. The reason is that the energy minimum belongs to the same spatial configurations, that is, to a triangular pyramid for $N=4$, for example 48.

In Table IX we show results for bosonic and fermionic systems with a purely attractive 
$G m^{2} / r$ ("gravitational") interaction. An $N$-body system of identical particles bound together by attractive pair potentials always collapses in large- $N$ limit (the binding energy per particle rises with $N$ to infinity), even if the particles are fermions. Self-gravitating boson systems have recently attracted some interest [49]. For these systems, both variational lower and upper bounds are available. In this case even the five-fermion system is bound. Thus the lack of bound states in five-electron-positron systems is a joint effect of the antisymmetry and of the repulsion between identical particles.

Finally, we mention an example involving an excited state. With $\mathcal{K}=500$, the SVM gives the energies of the ground and first excited states of the $t+d+\mu^{-}$system as -111.3640 and -100.9121 a.u., which are respectively compared to -111.364342 and -100.916421 a.u. of the CRCG result [50] with $\mathcal{K}=1442$, while the configuration-space Faddeev calculation [2] gives -111.36 a.u. for the ground state but no information for the excited state.

\section{SUMMARY}

We have formulated a variational calculation for few-body systems using the stochastic variational method on the correlated Gaussian basis. We have demonstrated the versatility of the correlated Gaussians and the efficiency of the stochastic variation by various numerical examples for $N=2-7$-particle systems. All the details of both formulation and calculational procedure are included to make this paper self-contained and easily reproducible.

The comparison with other calculations has corroborated the accuracy and efficiency of the method. In none of the test cases has the present method proved to be inferior to any of the alternative methods, and yet the method does not require excessive computational effort.

The correlation between the particles plays an important role in describing the fewbody system realistically. It has been taken into account in the framework of the correlated Gaussian functions. The correlated Gaussians are constructed from products of the Gaussian wave-packet single-particle functions through an integral transformation, which has enabled us to evaluate the center-of-mass motion free matrix elements analytically starting from the single-particle level. The nonlinear parameters of the correlated Gaussians have been selected by the stochastic variational method with a trial and error procedure. The success of the method using the correlated Gaussian basis is probably due to the fact that none of the Gaussians is indispensable, that is, there are different sets of the Gaussian parameters that represent the wave function equally well.

The method presented in this paper can be useful to solve few-body problems in diverse fields of physics such as description of microclusters, non-relativistic quark model, and halo nuclei. Among others, the most important application is the solution of the nuclear few-body problem, that is, a description of light nuclei by using realistic nucleon-nucleon potentials. In this case one has to take into account both short-range repulsion and higher orbital angular momenta required by the non-central components. Our test examples show that the correlated Gaussian basis function might be a suitable candidate to cope with these requirements. As is explained in Appendix B, the evaluation of the matrix elements for the non-central potentials poses no serious problem, and calculations including such potentials for nuclear few-body systems are under way. 
The limitations of the present method are those implied by the basis size and by the computer memory to store the matrix elements in the generator coordinate space. The limitations may become excessive as the number of particles and/or spin and isospin configurations become large.

To extend the method to nuclei of larger mass number in an approximate way, one can freeze part of the model space for a group of nucleons (cluster). One can omit, for example, some of the spin or isospin channels. It might be a good approximation to consider only those spin channels where the spins of the like nucleon pairs are coupled to zero. One can also restrict the intrinsic spatial motion of a cluster by fixing the nonlinear parameters to some appropriate values. One can introduce $N$ clusters and place the nucleons of each cluster into a common harmonic oscillator well, for example. The microscopic multicluster model is based on this approximation. The matrix elements needed in this multicluster model are given as a special case of those presented in this paper. In fact, one only needs to choose the single-particle generator coordinates, $\left(\mathbf{s}_{1}, \ldots, \mathbf{s}_{A}\right)$, such that

$$
\mathbf{s}_{n_{i-1}+1}=\mathbf{s}_{n_{i-1}+2}=\cdots=\mathbf{s}_{n_{i-1}+n_{i}} \quad(i=1, \ldots, N),
$$

where $n_{i}$ is the number of nucleons in the $i$ th cluster $\left(n_{0}=0, n_{1}+\cdots+n_{N}=A\right)$, and needs to couple appropriately the spins and isospins of the nucleons in each cluster to the spin and isospin of the cluster. The microscopic multicluster model has been successfully applied for description of the structure of light nuclei (see, for example, [17,22.23]).

Finally, we summarize some merits of our method in the following:

(i) Fully analytical calculational scheme; this plays a major role in the high speed and accuracy of the calculation.

(ii) Universality of the scheme. One needs to introduce no change, for example, between describing a multinucleon system and a Coulombic few-body system. It is easily adaptable to identical or non-identical particles, to fermions or bosons or mixed systems. The masses of particles may be different, yet no problem with the center-of-mass motion arises.

(iii) No expansion of the interaction is needed, and thus no problems in partial-wave truncation arise.

(iv) The convergence of the energy is fast. If one needs just a 2-3-digit estimate of the energy, it is enough to use a very small basis.

(v) The method is also accurate for excited states, which are obtained simultaneously with the same diagonalization (provided their angular momenta and parities are the same as those of the ground state; but only such excited states may be problematic).

(vi) The wave function is obtained in a compact, analytical form. It is then easy to use it in calculations of physical properties. It is "portable", reproducible, and easily testable. 


\section{APPENDIX A. SINGLE-PARTICLE MATRIX ELEMENTS}

The aim of this appendix is to list the single-particle matrix elements between Gaussian wave packets (Eq. (17)). The Gaussian packets are generalized in this appendix to have different width parameters and the expressions are therefore slightly more general than needed in the formulae of the main text. These single-particle matrix elements are, however, required for treating particles of unequal masses, in which the width parameter $\nu$ belonging to the particle of mass $m$ is to be chosen by Eq. (59). The overlap of two Gaussian wave packets is

$$
\left\langle\varphi_{\mathbf{s}_{1}}^{\nu_{1}} \mid \varphi_{\mathbf{s}_{2}}^{\nu_{2}}\right\rangle=\left(\frac{2 \sqrt{\nu_{1} \nu_{2}}}{\nu_{1}+\nu_{2}}\right)^{3 / 2} \exp \left(-\frac{\nu_{1} \nu_{2}}{\nu_{1}+\nu_{2}}\left(\mathbf{s}_{1}-\mathbf{s}_{2}\right)^{2}\right)
$$

The matrix element of the kinetic energy operator $\left(T=-\frac{\hbar^{2}}{2 M} \Delta\right)$ reads as

$$
\left\langle\varphi_{\mathbf{s}_{1}}^{\nu_{1}}|T| \varphi_{\mathbf{s}_{2}}^{\nu_{2}}\right\rangle=\frac{\hbar^{2}}{2 M} \frac{2 \nu_{1} \nu_{2}}{\nu_{1}+\nu_{2}}\left(3-\frac{2 \nu_{1} \nu_{2}}{\nu_{1}+\nu_{2}}\left(\mathbf{s}_{1}-\mathbf{s}_{2}\right)^{2}\right)\left\langle\varphi_{\mathbf{s}_{1}}^{\nu_{1}} \mid \varphi_{\mathbf{s}_{2}}^{\nu_{2}}\right\rangle
$$

The matrix element of the square radius becomes

$$
\left\langle\varphi_{\mathbf{s}_{1}}^{\nu_{1}}\left|\mathbf{r}_{1}^{2}\right| \varphi_{\mathbf{s}_{2}}^{\nu_{2}}\right\rangle=\frac{1}{2\left(\nu_{1}+\nu_{2}\right)}\left(3+\frac{2}{\nu_{1}+\nu_{2}}\left(\nu_{1} \mathbf{s}_{1}+\nu_{2} \mathbf{s}_{2}\right)^{2}\right)\left\langle\varphi_{\mathbf{s}_{1}}^{\nu_{1}} \mid \varphi_{\mathbf{s}_{2}}^{\nu_{2}}\right\rangle
$$

The two-particle matrix element of a $\delta$-function is given by

$$
\begin{aligned}
& \left\langle\varphi_{\mathbf{s}_{1}}^{\nu_{1}} \varphi_{\mathbf{s}_{2}}^{\nu_{2}}\left|\delta\left(\mathbf{r}_{1}-\mathbf{r}_{2}-\mathbf{r}\right)\right| \varphi_{\mathbf{s}_{3}}^{\nu_{3}} \varphi_{\mathbf{s}_{4}}^{\nu_{4}}\right\rangle=\left(\frac{\left(\nu_{1}+\nu_{3}\right)\left(\nu_{2}+\nu_{4}\right)}{\left(\nu_{1}+\nu_{2}+\nu_{3}+\nu_{4}\right) \pi}\right)^{3 / 2} \\
\times & \exp \left(-\frac{\left(\nu_{1}+\nu_{3}\right)\left(\nu_{2}+\nu_{4}\right)}{\nu_{1}+\nu_{2}+\nu_{3}+\nu_{4}}\left(\mathbf{r}-\frac{\nu_{1} \mathbf{s}_{1}+\nu_{3} \mathbf{s}_{3}}{\nu_{1}+\nu_{3}}+\frac{\nu_{2} \mathbf{S}_{2}+\nu_{4} \mathbf{s}_{4}}{\nu_{2}+\nu_{4}}\right)^{2}\right)\left\langle\varphi_{\mathbf{s}_{1}}^{\nu_{1}} \mid \varphi_{\mathbf{s}_{3}}^{\nu_{3}}\right\rangle\left\langle\varphi_{\mathbf{s}_{2}}^{\nu_{2}} \mid \varphi_{\mathbf{s}_{4}}^{\nu_{4}}\right\rangle .
\end{aligned}
$$

\section{APPENDIX B. MATRIX ELEMENTS OF THE TWO-BODY POTENTIALS}

The scope of this appendix is the calculation of the matrix elements of the different ingredients of the two-nucleon interaction between Gaussian wave packets. Most of the widely used coordinate-space two-nucleon interactions consist of central- $\left(O_{12}^{c}\right)$, tensor- $\left(O_{12}^{t}\right)$, spin-orbit- $\left(O_{12}^{b}\right), \mathbf{L}^{2}-\left(\right.$ or $\left.\mathbf{p}^{2}\right)\left(O_{12}^{q}\right)$ and quadratic spin-orbit $\left(O_{12}^{b b}\right)$ type potentials. (We follow

the abbreviated notations - $c, t, b, q, b b$ - invented by Urbana-Argonne group [51,52]. The definition of these operators is given in Table X.) These potential terms might be multiplied by the $\boldsymbol{\tau}_{1} \cdot \boldsymbol{\tau}_{2}\left(O_{12}^{\tau}\right), \boldsymbol{\sigma}_{1} \cdot \boldsymbol{\sigma}_{2}\left(O_{12}^{\sigma}\right)$, or $\boldsymbol{\tau}_{1} \cdot \boldsymbol{\tau}_{2} \boldsymbol{\sigma}_{1} \cdot \boldsymbol{\sigma}_{2}\left(O_{12}^{\tau \sigma}\right)$ spin-isospin operators and then one ends up with the general form

$$
V_{i j}=\sum_{p} \int d \mathbf{r} V^{p}(r) \delta\left(\mathbf{r}_{i}-\mathbf{r}_{j}-\mathbf{r}\right) O_{i j}^{p}
$$

where $p=c, c \tau, c \sigma, c \tau \sigma, t, t \tau, b, b \tau, q, q \tau, q \sigma, q \tau \sigma, b b, b b \tau$ is the short-hand notation to specify the component and $V^{p}(r)$ is the corresponding radial form factor. By using Eqs. (16), 
(17), (68), (69), and after some straightforward transformation, the matrix element of this interaction can be written as

$$
\begin{aligned}
& \left\langle\hat{\phi}_{\mathbf{s}_{1} \sigma_{1} \tau_{1}}^{\nu} \hat{\phi}_{\mathbf{s}_{2} \sigma_{2} \tau_{2}}^{\nu}\left|V_{12}\right| \hat{\phi}_{\mathbf{s}_{1}{ }^{\prime} \sigma_{1}^{\prime} \tau_{1}^{\prime}}^{\nu} \hat{\phi}_{\mathbf{s}_{2}{ }^{\prime} \sigma_{2}^{\prime} \tau_{2}^{\prime}}^{\nu}\right\rangle=\sum_{p} \int d \mathbf{r} V^{p}(r) f_{p}(r) \mathcal{M}(\mathbf{r}) \\
\times & \left\langle\chi_{\frac{1}{2} \sigma_{1}} \mathcal{X}_{\frac{1}{2} \tau_{1}} \chi_{\frac{1}{2} \sigma_{2}} \mathcal{X}_{\frac{1}{2} \tau_{2}}\left|r^{2} B^{p}+\sum_{l=0}^{2} \sum_{m=-l}^{l}(-1)^{m} r^{l} Y_{l m}(\hat{\mathbf{r}}) C_{l-m}^{p}\right| \chi_{\frac{1}{2} \sigma_{1}^{\prime}} \mathcal{X}_{\frac{1}{2} \tau_{1}^{\prime}} \chi_{\frac{1}{2} \sigma_{2}^{\prime}} \mathcal{X}_{\frac{1}{2} \tau_{2}^{\prime}}\right\rangle,
\end{aligned}
$$

with

$$
\mathcal{M}(\mathbf{r})=\left(\frac{\nu}{\pi}\right)^{3 / 2} \exp \left(-\nu r^{2}+\nu\left(\mathbf{s}_{1}-\mathbf{s}_{2}+\mathbf{s}_{1}^{\prime}-\mathbf{s}_{2}^{\prime}\right) \cdot \mathbf{r}-\frac{\nu}{4}\left(\mathbf{s}_{1}-\mathbf{s}_{2}+\mathbf{s}_{1}^{\prime}-\mathbf{s}_{2}^{\prime}\right)^{2}\right)\left\langle\varphi_{\mathbf{s}_{1}}^{\nu} \mid \varphi_{\mathbf{s}_{1}^{\prime}}^{\nu}\right\rangle\left\langle\varphi_{\mathbf{s}_{2}}^{\nu} \mid \varphi_{\mathbf{s}_{2}^{\prime}}^{\nu}\right\rangle .
$$

The $B^{p}$ and $C_{l m}^{p}$, independent of $\mathbf{r}$, are the operators in spin-isospin space and are listed in Table $\mathrm{X}$ for the most important terms $(c, t, b, q, b b)$. The remaining terms can be easily derived by multiplying these operators by the appropriate $\boldsymbol{\sigma}$ and $\boldsymbol{\tau}$ operators. The function $f_{p}(r)$ has the simple form: $f_{p}(r)=r^{-2}$ if $p=t$ or $t \tau$, and $f_{p}(r)=1$ otherwise.

The calculation of matrix elements of the operators appearing in Table $\mathrm{X}$ in the spin part is easily done with the Clebsch-Gordan coefficient. They are given below by suppressing the spin function $\chi_{\frac{1}{2} \sigma_{i}}\left(m_{i}=\frac{1}{2} \sigma_{i}\right)$.

$$
\begin{aligned}
& \left\langle m_{1} m_{2}\left|\boldsymbol{\sigma}_{1} \cdot \boldsymbol{\sigma}_{2}\right| m_{1}^{\prime} m_{2}^{\prime}\right\rangle \\
= & 3(-1)^{m_{1}-m_{1}^{\prime}} \delta_{m_{1}+m_{2}, m_{1}^{\prime}+m_{2}^{\prime}}\left\langle\frac{1}{2} m_{1}^{\prime} 1 m_{1}-m_{1}^{\prime} \mid \frac{1}{2} m_{1}\right\rangle\left\langle\frac{1}{2} m_{2}^{\prime} 1 m_{2}-m_{2}^{\prime} \mid \frac{1}{2} m_{2}\right\rangle . \\
& \left\langle m_{1} m_{2}\left|\left[\boldsymbol{\sigma}_{1} \times \boldsymbol{\sigma}_{2}\right]_{m}^{(2)}\right| m_{1}^{\prime} m_{2}^{\prime}\right\rangle=3 \delta_{m, m_{1}+m_{2}-m_{1}^{\prime}-m_{2}^{\prime}} \\
\times & \left\langle\frac{1}{2} m_{1}^{\prime} 1 m_{1}-m_{1}^{\prime} \mid \frac{1}{2} m_{1}\right\rangle\left\langle\frac{1}{2} m_{2}^{\prime} 1 m_{2}-m_{2}^{\prime} \mid \frac{1}{2} m_{2}\right\rangle\left\langle 1 m_{1}-m_{1}^{\prime} 1 m_{2}-m_{2}^{\prime} \mid 2 m\right\rangle . \\
& \left\langle m_{1} m_{2}\left|\left[\mathbf{x} \times\left(\boldsymbol{\sigma}_{1}+\boldsymbol{\sigma}_{2}\right)\right]_{m}^{(1)}\right| m_{1}^{\prime} m_{2}^{\prime}\right\rangle=\sqrt{3} \sum_{q_{1}, q_{2}=-1}(\mathbf{x})_{q_{1}} \\
\times & \left\langle 1 q_{1} 1 q_{2} \mid 1 m\right\rangle\left\{\delta_{m_{2}, m_{2}^{\prime}}\left\langle\frac{1}{2} m_{1}^{\prime} 1 q_{2} \mid \frac{1}{2} m_{1}\right\rangle+\delta_{m_{1}, m_{1}^{\prime}}\left\langle\frac{1}{2} m_{2}^{\prime} 1 q_{2} \mid \frac{1}{2} m_{2}\right\rangle\right\} . \\
& \left\langle m_{1} m_{2}\left|\left(\mathbf{x} \cdot \boldsymbol{\sigma}_{2}\right) \boldsymbol{\sigma}_{1_{m}}+\left(\mathbf{x} \cdot \boldsymbol{\sigma}_{1}\right) \boldsymbol{\sigma}_{2_{m}}\right| m_{1}^{\prime} m_{2}^{\prime}\right\rangle \\
= & 3\left\{(-1)^{m_{2}-m_{2}^{\prime}} \delta_{m, m_{1}-m_{1}^{\prime}}(\mathbf{x})_{m_{2}^{\prime}-m_{2}}+(-1)^{m_{1}-m_{1}^{\prime}} \delta_{m, m_{2}-m_{2}^{\prime}}(\mathbf{x})_{m_{1}^{\prime}-m_{1}}\right\} \\
\times & \left\langle\frac{1}{2} m_{1}^{\prime} 1 m_{1}-m_{1}^{\prime} \mid \frac{1}{2} m_{1}\right\rangle\left\langle\frac{1}{2} m_{2}^{\prime} 1 m_{2}-m_{2}^{\prime} \mid \frac{1}{2} m_{2}\right\rangle . \\
& \times(\mathbf{x})_{m_{1}^{\prime}-m_{1}}(\mathbf{x})_{m_{2}^{\prime}-m_{2}}\left\langle\frac{1}{2} m_{1}^{\prime} 1 m_{1}-m_{1}^{\prime} \mid \frac{1}{2} m_{1}\right\rangle\left\langle\frac{1}{2} m_{2}^{\prime} 1 m_{2}-m_{2}^{\prime} \mid \frac{1}{2} m_{2}\right\rangle . \\
& \left\langle m_{1} m_{2}\left|\left(\mathbf{x} \cdot \boldsymbol{\sigma}_{1}\right)\left(\mathbf{x} \cdot \boldsymbol{\sigma}_{2}\right)\right| m_{1}^{\prime} m_{2}^{\prime}\right\rangle=3(-1)^{m_{1}-m_{1}^{\prime}+m_{2}-m_{2}^{\prime}} \\
& \\
& \\
&
\end{aligned}
$$




$$
\begin{aligned}
& \left\langle m_{1} m_{2}\left|\left[\left[\mathbf{x} \times \boldsymbol{\sigma}_{1}\right]^{(1)} \times\left[\mathbf{x} \times \boldsymbol{\sigma}_{2}\right]^{(1)}\right]_{m}^{(2)}\right| m_{1}^{\prime} m_{2}^{\prime}\right\rangle \\
= & 3\left\langle\frac{1}{2} m_{1}^{\prime} 1 m_{1}-m_{1}^{\prime} \mid \frac{1}{2} m_{1}\right\rangle\left\langle\frac{1}{2} m_{2}^{\prime} 1 m_{2}-m_{2}^{\prime} \mid \frac{1}{2} m_{2}\right\rangle \\
\times & \sum_{q_{1}, q_{2}=-1}^{1}(\mathbf{x})_{q_{1}}(\mathbf{x})_{q_{2}}\left\langle 1 q_{1}+m_{1}-m_{1}^{\prime} 1 q_{2}+m_{2}-m_{2}^{\prime} \mid 2 m\right\rangle \\
\times & \left\langle 1 q_{1} 1 m_{1}-m_{1}^{\prime} \mid 1 q_{1}+m_{1}-m_{1}^{\prime}\right\rangle\left\langle 1 q_{2} 1 m_{2}-m_{2}^{\prime} \mid 1 q_{2}+m_{2}-m_{2}^{\prime}\right\rangle .
\end{aligned}
$$

Here $\mathbf{x}$ is a 3 -dimensional vector and $(\mathbf{x})_{m}$ stands for its spherical component $\sqrt{\frac{4 \pi}{3}} x Y_{1 m}(\hat{\mathbf{x}})$.

\section{APPENDIX C. MATRIX ELEMENTS OF SLATER DETERMINANTS}

In this appendix we briefly outline the calculation of the matrix elements between Slater determinants of Eq. (18) and show their concrete functional form, that is, the dependence on the generator coordinates s. We assume that the width parameter of the Gaussian wave packet is chosen to be the same for all nucleons. The overlap of two Slater determinants is equal to the determinant of the matrix of the single-particle overlaps:

$$
\left\langle\phi_{\kappa}\left(\mathbf{s}_{1}, \ldots, \mathbf{s}_{N}\right) \mid \phi_{\kappa^{\prime}}\left(\mathbf{s}_{1}^{\prime}, \ldots, \mathbf{s}_{N}^{\prime}\right)\right\rangle=\operatorname{det}\{B\}
$$

where

$$
B_{i j}=\left\langle\hat{\varphi}_{\mathbf{s}_{i} \sigma_{i} \tau_{i}}^{\nu} \mid \hat{\varphi}_{\mathbf{s}^{\prime} \sigma_{j}^{\prime} \tau_{j}^{\prime}}^{\nu}\right\rangle \quad(i, j=1, \ldots, N)
$$

By using the definition of the determinant, this can be rewritten as

$$
\left\langle\phi_{\kappa}\left(\mathbf{s}_{1}, \ldots, \mathbf{s}_{N}\right) \mid \phi_{\kappa^{\prime}}\left(\mathbf{s}_{1}^{\prime}, \ldots, \mathbf{s}_{N}^{\prime}\right)\right\rangle=\sum_{P}^{N !} \operatorname{sign}(P)\left\langle\hat{\varphi}_{\mathbf{s}_{1} \sigma_{1} \tau_{1}}^{\nu} \mid \hat{\varphi}_{\mathbf{s}^{\prime} p_{1} \sigma_{p_{1}}^{\prime} \tau_{p_{1}}^{\prime}}^{\nu}\right\rangle \ldots\left\langle\hat{\varphi}_{\mathbf{s}_{N} \sigma_{N} \tau_{N}}^{\nu} \mid \hat{\varphi}_{\mathbf{s}_{p_{N}} \sigma_{p_{N}}^{\prime} \tau_{p_{N}}^{\prime}}^{\nu}\right\rangle,
$$

where $\left(p_{1} \cdots p_{N}\right)$ is the permutation $P$ of the set $(1 \cdots N)$. Substituting the overlap of the single-particle overlaps of Eq. (65) into Eq. (80) yields an explicit formula for the overlap of the Slater determinants:

$$
\left\langle\phi_{\kappa}\left(\mathbf{s}_{1}, \ldots, \mathbf{s}_{N}\right) \mid \phi_{\kappa^{\prime}}\left(\mathbf{s}_{1}^{\prime}, \ldots, \mathbf{s}_{N}^{\prime}\right)\right\rangle=\sum_{P}^{N !} C_{P} \mathrm{e}^{-\frac{1}{2} \tilde{\mathbf{s}} A_{P} \mathbf{s}},
$$

where the matrix $A_{P}$ is defined by

$$
\left(A_{P}\right)_{i j}=\left(A_{P}\right)_{N+i, N+j}=\nu \delta_{i j}, \quad\left(A_{P}\right)_{i, N+j}=\left(A_{P}\right)_{N+j, i}=-\nu \delta_{j, p_{i}} \quad(1 \leq i, j \leq N),
$$

and

$$
C_{P}=\operatorname{sign}(P) \delta_{\sigma_{1} \sigma^{\prime} p_{1}} \delta_{\tau_{1} \tau^{\prime} p_{1}} \cdots \delta_{\sigma_{N} \sigma^{\prime} p_{N}} \delta_{\tau_{N} \tau^{\prime} p_{N}} .
$$

The orthogonality of the spin-isospin functions greatly reduces the number of terms in the summation over $P$.

The matrix element of the kinetic energy operator is 


$$
\sum_{i=1}^{N}\left\langle\phi_{\kappa}\left(\mathbf{s}_{1}, \ldots, \mathbf{s}_{N}\right)\left|T_{i}\right| \phi_{\kappa^{\prime}}\left(\mathbf{s}_{1}^{\prime}, \ldots, \mathbf{s}_{N}^{\prime}\right)\right\rangle=\sum_{i=1}^{N} \sum_{j=1}^{N}\left\langle\hat{\varphi}_{\mathbf{s}_{i} \sigma_{i} \tau_{i}}^{\nu}|T| \hat{\varphi}_{\mathbf{s}_{j}^{\prime} \sigma_{j}^{\prime} \tau_{j}^{\prime}}^{\nu}\right\rangle(-1)^{i+j} \operatorname{det}\left\{B^{i j}\right\},
$$

where $B^{i j}$ is obtained by omitting the $i$ th row and the $j$ th column of the matrix $B$ defined in Eq. (79). The substitution of the single-particle overlaps and the single-particle matrix elements of the kinetic energy operator enables us to obtain

$$
\sum_{i=1}^{N}\left\langle\phi_{\kappa}\left(\mathbf{s}_{1}, \ldots, \mathbf{s}_{N}\right)\left|T_{i}\right| \phi_{\kappa^{\prime}}\left(\mathbf{s}^{\prime}{ }_{1}, \ldots, \mathbf{s}_{N}^{\prime}\right)\right\rangle=\frac{\hbar \omega}{2} \sum_{P}^{N !} C_{P}\left(\frac{3}{2} N-\frac{1}{2} \tilde{\mathbf{s}} A_{P} \mathbf{s}\right) \mathrm{e}^{-\frac{1}{2} \tilde{\mathbf{s}} A_{P} \mathbf{s}} .
$$

Note that the coefficients $C_{P}$ and the matrices $A_{P}$ in Eqs. (81) and (85) are the same. The manipulation similar to this leads us to the matrix element of the square radius as

$$
\sum_{i=1}^{N}\left\langle\phi_{\kappa}\left(\mathbf{s}_{1}, \ldots, \mathbf{s}_{N}\right)\left|\mathbf{r}_{i}^{2}\right| \phi_{\kappa^{\prime}}\left(\mathbf{s}^{\prime}, \ldots, \mathbf{s}_{N}^{\prime}\right)\right\rangle=\frac{1}{2 \nu} \sum_{P}^{N !} C_{P}\left(\frac{3}{2} N-\frac{1}{2} \tilde{\mathbf{s}} A_{P} \mathbf{s}+\nu \tilde{\mathbf{s}} \mathbf{s}\right) \mathrm{e}^{-\frac{1}{2} \tilde{\mathbf{s}} A_{P} \mathbf{s}} .
$$

As is explained in Appendix B, the matrix element of any two-body interaction between the Slater determinants may be reduced to the following:

$$
\sum_{i<j}^{N}\left\langle\phi_{\kappa}\left(\mathbf{s}_{1}, \ldots, \mathbf{s}_{N}\right)\left|\delta\left(\mathbf{r}_{i}-\mathbf{r}_{j}-\mathbf{r}\right) O_{i j}^{p}\right| \phi_{\kappa^{\prime}}\left(\mathbf{s}^{\prime}{ }_{1}, \ldots, \mathbf{s}_{N}^{\prime}\right)\right\rangle .
$$

The matrix element of Eq. (87) is called the correlation function of type $p$ evaluated between the Slater determinants. The calculation of this matrix element can be done with the use of the basic two-body matrix elements of Appendix B and the single-particle overlaps. We will show, as an example, the case of $O_{i j}^{p}=1$, i.e., Wigner-type $\delta$-function two-body interaction $(p=c)$. Then we have

$$
\begin{aligned}
& \sum_{i<j}^{N}\left\langle\phi_{\kappa}\left(\mathbf{s}_{1}, \ldots, \mathbf{s}_{N}\right)\left|\delta\left(\mathbf{r}_{i}-\mathbf{r}_{j}-\mathbf{r}\right)\right| \phi_{\kappa^{\prime}}\left(\mathbf{s}_{1}^{\prime}, \ldots, \mathbf{s}_{N}^{\prime}\right)\right\rangle \\
= & \sum_{i<j}^{N} \sum_{k, l=1}^{N}\left\langle\hat{\varphi}_{\mathbf{s}_{i} \sigma_{i} \tau_{i}}^{\nu} \hat{\varphi}_{\mathbf{s}_{j} \sigma_{j} \tau_{j}}^{\nu}\left|\delta\left(\mathbf{r}_{i}-\mathbf{r}_{j}-\mathbf{r}\right)\right| \hat{\varphi}_{\mathbf{s}_{k}^{\prime} \sigma_{k}^{\prime} \tau_{k}}^{\prime} \hat{\varphi}_{\mathbf{s}^{\prime} l \sigma_{l}^{\prime} \tau_{l}^{\prime}}^{\nu}\right\rangle(-1)^{i+j+k+l} \operatorname{det}\left\{B^{i j k l}\right\},
\end{aligned}
$$

where $B^{i j k l}$ is obtained by omitting the $i$ th and $j$ th rows and the $k$ th and $l$ th columns of the matrix $B$. By substituting the explicit formula of the ingredients, we arrive at the same form as given in Eq. (25):

$$
\begin{aligned}
& \sum_{i<j}^{N}\left\langle\phi_{\kappa}\left(\mathbf{s}_{1}, \ldots, \mathbf{s}_{N}\right)\left|\delta\left(\mathbf{r}_{i}-\mathbf{r}_{j}-\mathbf{r}\right)\right| \phi_{\kappa^{\prime}}\left(\mathbf{s}^{\prime}{ }_{1}, \ldots, \mathbf{s}_{N}^{\prime}\right)\right\rangle \\
= & \left(\frac{\nu}{\pi}\right)^{3 / 2} \mathrm{e}^{-\nu r^{2}} \sum_{P}^{N !} C_{P} \sum_{i<j}^{N} \mathrm{e}^{-\frac{1}{2} \tilde{\mathbf{s}}\left(A_{P}+B_{P}^{(i j)}\right) \mathbf{s}+\mathbf{d}_{P}^{(i j)} \cdot \mathbf{r}},
\end{aligned}
$$

with

$$
\mathbf{d}_{P}^{(i j)}=\nu\left(\mathbf{s}_{i}-\mathbf{s}_{j}+\mathbf{s}_{N+p_{i}}-\mathbf{s}_{N+p_{j}}\right) .
$$


The coefficients $C_{P}$ and the matrices $A_{P}$ are the same as those that appear in Eq. (81). The matrix $B_{P}^{(i j)}$ is defined by

$$
\left(B_{P}^{(i j)}\right)_{k l}=\left(B_{P}^{(i j)}\right)_{l k}= \begin{cases}\frac{\nu}{2} & (k l)=(i i),(j j),\left(N+p_{i}, N+p_{i}\right),\left(N+p_{j}, N+p_{j}\right), \\ & \left(i, N+p_{i}\right),\left(j, N+p_{j}\right) . \\ -\frac{\nu}{2} & (k l)=(i j),\left(i, N+p_{j}\right),\left(j, N+p_{i}\right),\left(N+p_{i}, N+p_{j}\right) . \\ 0 & \text { otherwise. }\end{cases}
$$

\section{APPENDIX D. EVALUATION OF THE OVERLAP OF THE CORRELATED GAUSSIANS}

In this appendix we derive the overlap of the basis functions, defined in Eqs. (2) and (6), to illustrate the calculation of the matrix elements through the operations prescribed in Eq. (57). For the sake of simplicity we work out the case of overlap only, but the matrix elements of the kinetic and the potential energy are not much more involved either and these matrix elements can be calculated by repeating the steps detailed here.

In Eq. (57) the matrix element of an operator $\mathcal{O}$ between the correlated Gaussians are derived from the matrix elements between the Gaussian packets by applying suitably chosen operations. In the case of $\mathcal{O}=1$, upon substituting the matrix elements in the generator coordinate space (Eq. (41)), Eq. (57) reads as

$$
\begin{aligned}
& \left\langle\mathcal{A}\left\{f_{K L M}(\mathbf{u}, \mathbf{x}, A) \chi_{S M_{S}} \mathcal{X}_{T M_{T}}\right\} \mid \mathcal{A}^{\prime}\left\{f_{K^{\prime} L^{\prime} M^{\prime}}\left(\mathbf{u}^{\prime}, \mathbf{x}, A^{\prime}\right) \chi_{S^{\prime} M_{S}^{\prime}} \mathcal{X}_{T^{\prime} M_{T}^{\prime}}\right\}\right\rangle \\
\sim & \iint d \hat{\mathbf{t}} d \hat{\mathbf{t}}^{\prime} Y_{L M}(\hat{\mathbf{t}})^{*} Y_{L^{\prime} M^{\prime}}\left(\hat{\mathbf{t}}^{\prime}\right) \\
\times & \left(\frac{d^{\kappa+\kappa^{\prime}}}{d \alpha^{\kappa} d \alpha^{\prime \kappa^{\prime}}} \mathrm{e}^{-\frac{1}{2} \tilde{\mathbf{T}} C \mathbf{T}} \int d \mathbf{S} \mathrm{e}^{-\frac{1}{2} \tilde{\mathbf{S}} Q \mathbf{S}+\tilde{\mathbf{T}} \mathbf{S}} \sum_{i=1}^{n_{o}} C_{i}^{(o)} \mathrm{e}^{-\frac{1}{2} \tilde{\mathbf{S}} B_{i}^{(o)} \mathbf{S}}\right)_{\substack{\alpha=\alpha^{\prime}=0 \\
t=t^{\prime}=1}},
\end{aligned}
$$

with

$$
\kappa=2 K+L, \quad \kappa^{\prime}=2 K^{\prime}+L^{\prime},
$$

where a constant factor is omitted for a moment. By integrating over the generator coordinates $\mathbf{S}$ with the use of Eq. (52), one readily obtains

$$
\begin{aligned}
& \left\langle\mathcal{A}\left\{f_{K L M}(\mathbf{u}, \mathbf{x}, A) \chi_{S M_{S}} \mathcal{X}_{T M_{T}}\right\} \mid \mathcal{A}^{\prime}\left\{f_{K^{\prime} L^{\prime} M^{\prime}}\left(\mathbf{u}^{\prime}, \mathbf{x}, A^{\prime}\right) \chi_{S^{\prime} M_{S}^{\prime}} \mathcal{X}_{T^{\prime} M_{T}^{\prime}}\right\}\right\rangle \\
\sim & \iint d \hat{\mathbf{t}} d \hat{\mathbf{t}}^{\prime} Y_{L M}(\hat{\mathbf{t}})^{*} Y_{L^{\prime} M^{\prime}}\left(\hat{\mathbf{t}}^{\prime}\right) \\
\times & \left(\frac{d^{\kappa+\kappa^{\prime}}}{d \alpha^{\kappa} d \alpha^{\prime \kappa^{\prime}}} \sum_{i=1}^{n_{o}} C_{i}^{(o)}\left(\frac{(2 \pi)^{(2 N-2)}}{\operatorname{det}\left(B_{i}^{(o)}+Q\right)}\right)^{3 / 2} \mathrm{e}^{p_{i} \alpha^{2}+p_{i}{ }^{\prime} \alpha^{\prime 2}+q_{i} \alpha \alpha^{\prime} \mathbf{t} \cdot \mathbf{t}^{\prime}}\right)_{\substack{\alpha=\alpha^{\prime}=0 \\
t=t^{\prime}=1}},
\end{aligned}
$$

where, by using Eqs. (54) and (55), we introduced the abbreviations

$$
\begin{aligned}
p_{i} & =\frac{1}{2} \sum_{j, k=1}^{N-1} w_{j}\left(\left(B_{i}^{(o)}+Q\right)^{-1}-C\right)_{j k} w_{k}, \quad p_{i}{ }^{\prime}=\frac{1}{2} \sum_{j, k=N}^{2 N-2} w_{j}{ }^{\prime}\left(\left(B_{i}^{(o)}+Q\right)^{-1}-C\right)_{j k} w_{k}{ }^{\prime}, \\
q_{i} & =\sum_{j=1}^{N-1} \sum_{k=N}^{2 N-2} w_{j}\left(\left(B_{i}^{(o)}+Q\right)^{-1}-C\right)_{j k} w_{k}{ }^{\prime},
\end{aligned}
$$


with

$$
w_{k}=\sum_{j=1}^{N-1}(\Gamma C)_{k j}^{-1} u_{j}, \quad w_{k}{ }^{\prime}=\sum_{j=N}^{2 N-2}\left(\Gamma C^{\prime}\right)_{k j}^{-1} u_{j}{ }^{\prime},
$$

in order to emphasize the $\alpha$ - and $\alpha^{\prime}$-dependence of the resulting expression. The differentiation with respect to $\alpha$ and $\alpha^{\prime}$ can be promptly given by expanding the exponential functions into power series:

$$
\begin{aligned}
& \frac{d^{\kappa+\kappa^{\prime}}}{d \alpha^{\kappa} d \alpha^{\prime \kappa^{\prime}}} \mathrm{e}^{p_{i} \alpha^{2}+p_{i}{ }^{\prime} \alpha^{2}+q_{i} \alpha \alpha^{\prime} \mathbf{t} \cdot \mathbf{t}^{\prime}} \\
= & \sum_{j, j^{\prime}, k}^{\infty} \frac{p_{i}^{j} p_{i}{ }^{\prime j^{\prime}}\left(q_{i} \mathbf{t} \cdot \mathbf{t}^{\prime}\right)^{k}}{j ! j^{\prime} ! k !} \frac{(2 j+k) !}{(2 j+k-\kappa) !} \frac{\left(2 j^{\prime}+k\right) !}{\left(2 j^{\prime}+k-\kappa^{\prime}\right) !} \alpha^{2 j+k-\kappa} \alpha^{2 j^{\prime}+k-\kappa^{\prime}}
\end{aligned}
$$

and therefore, after putting $\alpha=\alpha^{\prime}=0$, the calculation of Eq. (94) can be continued as

$$
\begin{aligned}
& \left\langle\mathcal{A}\left\{f_{K L M}(\mathbf{u}, \mathbf{x}, A) \chi_{S M_{S}} \mathcal{X}_{T M_{T}}\right\} \mid \mathcal{A}^{\prime}\left\{f_{K^{\prime} L^{\prime} M^{\prime}}\left(\mathbf{u}^{\prime}, \mathbf{x}, A^{\prime}\right) \chi_{S^{\prime} M_{S}^{\prime}} \mathcal{X}_{T^{\prime} M_{T}^{\prime}}\right\}\right\rangle \\
\sim & \iint d \hat{\mathbf{t}} d \hat{\mathbf{t}}^{\prime} Y_{L M}(\hat{\mathbf{t}})^{*} Y_{L^{\prime} M^{\prime}}\left(\hat{\mathbf{t}}^{\prime}\right) \\
\times & \left(\sum_{i=1}^{n_{o}} C_{i}^{(o)}\left(\frac{(2 \pi)^{2 N-2}}{\operatorname{det}\left(B_{i}^{(o)}+Q\right)}\right)^{3 / 2} \kappa ! \kappa^{\prime} ! \sum_{k} \frac{p_{i}^{\frac{\kappa-k}{2}} p_{i}{ }^{\frac{\kappa^{\prime}-k}{2}} q_{i}^{k}}{\left(\frac{\kappa-k}{2}\right) !\left(\frac{\kappa^{\prime}-k}{2}\right) ! k !}\left(\mathbf{t} \cdot \mathbf{t}^{\prime}\right)_{t=t^{\prime}=1}^{k}\right),
\end{aligned}
$$

where the summation over $k$ runs from 0 to $\max \left(\kappa, \kappa^{\prime}\right)$ for those values that fulfill $(-1)^{k+\kappa}=1$ and $(-1)^{k+\kappa^{\prime}}=1$.

The last step, the integration over the angles of $\mathbf{t}$ and $\mathbf{t}^{\prime}$, can be accomplished by applying Eq. (49) to express the scalar product $\mathbf{t} \cdot \mathbf{t}^{\prime}$, and then we get the final expression:

$$
\begin{aligned}
& \left\langle\mathcal{A}\left\{f_{K L M}(\mathbf{u}, \mathbf{x}, A) \chi_{S M_{S}} \mathcal{X}_{T M_{T}}\right\} \mid \mathcal{A}^{\prime}\left\{f_{K^{\prime} L^{\prime} M^{\prime}}\left(\mathbf{u}^{\prime}, \mathbf{x}, A^{\prime}\right) \chi_{S^{\prime} M_{S}^{\prime}} \mathcal{X}_{T^{\prime} M_{T}^{\prime}}\right\}\right\rangle \\
= & \delta_{L L^{\prime}} \delta_{M M^{\prime}} \frac{1}{B_{K L} B_{K^{\prime} L^{\prime}}^{\prime}}\left(\frac{(\operatorname{det} \Gamma)^{3}}{(4 \pi)^{(N-1)} \operatorname{det}(\Gamma-A) \operatorname{det}\left(\Gamma-A^{\prime}\right)}\right)^{3 / 2} \\
\times & \left(\sum_{i=1}^{n_{o}} C_{i}^{(o)}\left(\frac{(2 \pi)^{2 N-2}}{\operatorname{det}\left(B_{i}^{(o)}+Q\right)}\right)^{3 / 2} \kappa ! \kappa^{\prime} ! \sum_{k} \frac{p_{i}^{\frac{\kappa-k}{2}} p_{i}{ }^{\frac{\kappa^{\prime}-k}{2}} q_{i}^{k}}{\left(\frac{\kappa-k}{2}\right) !\left(\frac{\kappa^{\prime}-k}{2}\right) ! k !} B_{k L}\right)
\end{aligned}
$$

where the constant term previously suppressed is also included. The orthogonality of the matrix element in the spin and isospin quantum numbers is implicitly contained in the coefficients $C_{i}^{(o)}$.

The calculation of the matrix elements in the way described above is very simple. This fact becomes especially evident if one compares the work described above to the formidable task of the calculation of the matrix elements in the case where the function $\theta_{L M_{L}}(\mathbf{x})$ is decomposed into partial waves of the relative coordinates as in Eq. (5). In fact, in that latter case one has to integrate over the angles of the relative coordinates and one has to cope with complicated angular momentum algebra. We note, however, that the calculation of the matrix element of the latter type poses no problem if the function $\theta_{L M_{L}}(\mathbf{x})$ of Eq. (5) is expressed as a linear combination of the terms of Eq. (6) with appropriate $\mathbf{u}$-vectors. 
All the matrix elements can be given in a similar closed analytic form and the numerical evaluation of the matrix elements as a function of the nonlinear parameters is therefore straightforward. The values $\kappa=2 K+L$ and $\kappa^{\prime}=2 K^{\prime}+L^{\prime}$ are usually small in practical cases and the summation over $k$ is limited to just a few terms in Eq. (99).

This work was supported by OTKA grant No. T17298 (Hungary) and by a Grant-in-Aid for Scientific Research (No. 05243102 and No. 06640381) of the Ministry of Education, Science and Culture (Japan). K. V. gratefully acknowledges the hospitality of the RIKEN LINAC Laboratory and the support of the Science and Technology Agency of Japan, 1994. The authors are grateful for the use of RIKEN's VPP500 computer which made possible most of the calculations. Thanks are also due to the support of both Japan Society for the Promotion of Science and Hungarian Academy of Sciences, 1994-1995. The authors thank Prof. R. G. Lovas for careful reading of the manuscript and useful suggestions. 


\section{REFERENCES}

[1] J. L. Friar, B. F. Gibson, and G. L. Payne, Phys. Rev. C24, 2279 (1981).

[2] N. W. Schellingerhout and L. P. Kok, Nucl. Phys. A508, 299c (1990).

[3] W. Glöckle and H. Kamada, Phys. Rev. Lett. 71, 971 (1993).

[4] Y. Akaishi, in International Review of Nuclear Physics, Vol. 4, (World Scientific, Singapore, 1986), p.259.

[5] A. Kievsky, M. Viviani, and S. Rosati, Nucl. Phys. A501, 503 (1989); ibid. A551, 241 (1993); ibid. A577, 511 (1994).

[6] M. Kamimura and H. Kameyama, Nucl. Phys. A508 17c, (1990); H. Kameyama, M. Kamimura, and Y. Fukushima, Phys. Rev. C40, 974, (1989).

[7] J. Carlson and V. R. Pandharipande, Nucl. Phys. A371, 301 (1981).

[8] R. B. Wiringa, Nucl. Phys. A543, 199c (1992).

[9] J. G. Zabolitzky and M. H. Kalos, Nucl. Phys. A356, 114 (1981); J. G. Zabolitzky, K. E. Schmidt, and M. H. Kalos, Phys. Rev. C25, 1111 (1982).

[10] J. Carlson, Phys. Rev. C36, 2026 (1987).

[11] B. S. Pudliner, V. R. Pandharipande, J. Carlson, and R. B. Wiringa, Phys. Rev. Lett. 74, 4396 (1995).

[12] V. I. Kukulin and V. M. Krasnopol'sky, J. Phys. G3, 795 (1977).

[13] K. Varga, Y. Suzuki, and R. G. Lovas, Nucl. Phys. A371, 447 (1994).

[14] S. F. Boys, Proc. R. Soc. London Ser. A258, 402 (1960); K. Singer, ibid. A258, 412 (1960).

[15] S. A. Alexander, H. J. Monkhorst, and K. Szalewicz, J. Chem. Phys. 85, 5821 (1986).

[16] S. Fantoni, L. Panattoni, and S. Rosati, Nouvo Cimento A69, 81 (1970).

[17] K. Varga, Y. Suzuki, and Y. Ohbayasi, Phys. Rev. C50, 189 (1994); K. Arai, Y. Suzuki, and K. Varga, ibid. C51, 2488 (1995); K. Varga, Y. Suzuki, and I. Tanihata, submitted to Phys. Rev. C.

[18] P. M. Kozlowski and L. Adamowicz, J. Chem. Phys. 95, 6681 (1991).

[19] D. B. Kinghorn and R. D. Poshusta, Phys. Rev. A47, 3671 (1993).

[20] H. De Raedt and M. Frick, Phys. Rep. 231, 109 (1993).

[21] Y. Suzuki, E. J. Reske, and K. T. Hecht, Nucl. Phys. A381, 77 (1982).

[22] D. Brink, Proceedings of the International School of Physics "Enrico Fermi", Course $\mathbf{3 6}$ (1965), ed. C. Bloch, (Academic Press, New York and London, 1966), p.247.

[23] K. Wildermuth and Y. C. Tang, A Unified Theory of the Nucleus, Clustering Phenomena in Nuclei, Vol. 1, eds. K. Wildermuth and P. Kramer (Vieweg, Braunschweig, Germany, 1977).

[24] H. Horiuchi, Prog. Theor. Phys. Suppl. No. 62, 90 (1977).

[25] H. Feldmeier, Nucl. Phys. A515, 147 (1990).

[26] A. Ono, H. Horiuchi, T. Maruyama, and A. Ohnishi, Phys. Rev. C47, 2652 (1993).

[27] P. O. Löwdin, Phys. Rev. 97, 1490 (1955).

[28] M. Kamimura, Prog. Theor. Phys. Suppl. No. 62, 236 (1977).

[29] R. A. Malfliet and J. A. Tjon, Nucl. Phys. A127, 161 (1969).

[30] A. B. Volkov, Nucl. Phys. 74, 33 (1965).

[31] I. R. Afnan and Y. C. Tang, Phys. Rev. 175, 1337 (1968). 
[32] D. R. Thompson, M. LeMere, and Y. C. Tang, Nucl. Phys. A286, 53 (1977); I. Reichstein and Y. C. Tang, Nucl. Phys. A158, 529 (1970).

[33] H. Kamada and W. Glöckle, Nucl. Phys. A548, 205 (1992).

[34] R. B. Wiringa, private communication.

[35] G. Erens, J. L. Visschers, and R. van Wageningen, Ann. Phys. (N. Y.) 67, 461 (1971).

[36] J. L. Ballot, Z. Phys. A-Atoms and Nuclei 302, 347 (1981).

[37] J. L. Friar, quoted in [10].

[38] R. G. Lovas, A. T. Kruppa, R. Beck, and F. Dickmann, Nucl. Phys. A474 451, (1987).

[39] S. Ali and A. R. Bodmer, Nucl. Phys. 80, 99 (1966).

[40] J. A. Wheeler, Ann. N. Y. Acad. Sci. 48, 219 (1946).

[41] A. Yeremin, A. M. Frolov, and E. B. Kutukova, Few-Body Systems 4, 111 (1988).

[42] Y. K. Ho, J. Phys. B16, 1503 (1983).

[43] A. P. Mills, Phys. Rev. Lett. 46, 717 (1981).

[44] E. A. Hylleraas and A. Ore, Phys. Rev. 71, 493 (1947); A. Ore, ibid. 71, 913 (1947); ibid. 83, 665 (1951).

[45] A. M. Frolov, S. I. Kryuchkov, and V. H. Smith, Jr., Phys. Rev. A51, 4514 (1995).

[46] P. M. Kozlowski and L. Adamowicz, Phys. Rev. A47, 1903 (1993).

[47] S. Fleck and J.-M. Richard, Few-body systems, in press.

[48] Y. K. Ho, Phys. Rev. A33, 3584 (1986).

[49] J. L. Basdevant, A. Martin, and J. M. Richard, Nucl. Phys. B343, 60 (1990).

[50] M. Kamimura, Phys. Rev. A38, 621 (1988).

[51] I. E. Lagaris and V. R. Pandharipande, Nucl. Phys. A539, 331 (1981).

[52] R. B. Wiringa, R. A. Smith, and T. L. Ainsworth, Phys. Rev. C29, 1207 (1984). 


\section{TABLES}

TABLE I. List of the parameters of the nucleon-nucleon potentials used in this paper. The potential consists of a few terms; each is expressed as $V_{i} f\left(\mu_{i}, r\right)\left(W_{i}+B_{i} P_{\sigma}-H_{i} P_{\tau}+M_{i} P_{r}\right)$, where $P_{\sigma}, P_{\tau}$ and $P_{r}$ are the spin-, isospin- and space-exchange operators. The form factor $f\left(\mu_{i}, r\right)$ is $\exp \left(-\mu r^{2}\right)$ for the Gaussian potential or $\exp (-\mu r) / r$ for the Yukawa potential. The potential strength $V$ is in units of $\mathrm{MeV}$ and the range $\mu$ in units of $\mathrm{fm}^{-2}$ for Gaussian or $\mathrm{fm}^{-1}$ for Yukawa, respectively. The Majorana mixture $M$ of the Volokov potential is set to zero in the calculation. The Minnesota potential contains the parameter $u$, which is set to unity in the calculation.

\begin{tabular}{|c|c|c|c|c|c|c|c|c|}
\hline \hline \hline Potential & Type & $i$ & $V_{i}$ & $\mu_{i}$ & $W_{i}$ & $M_{i}$ & $B_{i}$ & $H_{i}$ \\
\hline MT-V & Yukawa & 1 & 1458.05 & 3.11 & 1.0 & 0.0 & 0.0 & 0.0 \\
[29] & & 2 & -578.09 & 1.55 & 1.0 & 0.0 & 0.0 & 0.0 \\
\hline Volkov & Gauss & 1 & 144.86 & $0.82^{-2}$ & $1.0-M$ & $M$ & 0.0 & 0.0 \\
[30] & & 2 & -83.34 & $1.60^{-2}$ & $1.0-M$ & $M$ & 0.0 & 0.0 \\
\hline ATS3 & Gauss & 1 & 1000.0 & 3.0 & 1.0 & 0.0 & 0.0 & 0.0 \\
[31] & & 2 & -326.7 & 1.05 & 0.5 & 0.0 & 0.5 & 0.0 \\
& & 3 & -166.0 & 0.80 & 0.5 & 0.0 & -0.5 & 0.0 \\
& & 4 & -43.0 & 0.60 & 0.5 & 0.0 & 0.5 & 0.0 \\
& & 5 & -23.0 & 0.40 & 0.5 & 0.0 & -0.5 & 0.0 \\
\hline Minnesota & Gauss & 1 & 200.0 & 1.487 & $0.5 u$ & $1.0-0.5 u$ & 0.0 & 0.0 \\
[32] & & 2 & -178.0 & 0.639 & $0.25 u$ & $0.5-0.25 u$ & $0.25 u$ & $0.5-0.25 u$ \\
& & 3 & -91.85 & 0.465 & $0.25 u$ & $0.5-0.25 u$ & $-0.25 u$ & $-0.5+0.25 u$ \\
\hline \hline
\end{tabular}


TABLE II. Spin (isospin) couplings of the nucleons. The possible intermediate spin (isospin) configurations of an $N$-nucleon system are listed above the $(N-1)$ th horizontal line. The spins of a 5-nucleon system, for example, are given by the first ten elements of the 5th column. The spins of the $2-3-4$ - particle subsystems of the 5-nucleon system is given in the first ten elements of the $1-2-3$ columns.

\begin{tabular}{|c|c|c|c|c|c|c|}
\hline \multirow[t]{21}{*}{$N$} & 2 & 3 & 4 & 5 & 6 & 7 \\
\hline & 0 & $1 / 2$ & 0 & $1 / 2$ & 0 & $1 / 2$ \\
\hline & 1 & $1 / 2$ & 0 & $1 / 2$ & 0 & $1 / 2$ \\
\hline & 1 & $3 / 2$ & 1 & $1 / 2$ & 0 & $1 / 2$ \\
\hline & 0 & $1 / 2$ & 1 & $1 / 2$ & 0 & $1 / 2$ \\
\hline & 1 & $1 / 2$ & 1 & $1 / 2$ & 0 & $1 / 2$ \\
\hline & 1 & $3 / 2$ & 2 & $3 / 2$ & 1 & $1 / 2$ \\
\hline & 1 & $3 / 2$ & 1 & $3 / 2$ & 1 & $1 / 2$ \\
\hline & 0 & $1 / 2$ & 1 & $3 / 2$ & 1 & $1 / 2$ \\
\hline & 1 & $1 / 2$ & 1 & $3 / 2$ & 1 & $1 / 2$ \\
\hline & 1 & $3 / 2$ & 2 & $5 / 2$ & 2 & $3 / 2$ \\
\hline & 0 & $1 / 2$ & 0 & $1 / 2$ & 1 & $1 / 2$ \\
\hline & 1 & $1 / 2$ & 0 & $1 / 2$ & 1 & $1 / 2$ \\
\hline & 1 & $3 / 2$ & 1 & $1 / 2$ & 1 & $1 / 2$ \\
\hline & 0 & $1 / 2$ & 1 & $1 / 2$ & 1 & $1 / 2$ \\
\hline & 1 & $1 / 2$ & 1 & $1 / 2$ & 1 & $1 / 2$ \\
\hline & 1 & $3 / 2$ & 2 & $3 / 2$ & 2 & $3 / 2$ \\
\hline & 1 & $3 / 2$ & 1 & $3 / 2$ & 2 & $3 / 2$ \\
\hline & 0 & $1 / 2$ & 1 & $3 / 2$ & 2 & $3 / 2$ \\
\hline & 1 & $1 / 2$ & 1 & $3 / 2$ & 2 & $3 / 2$ \\
\hline & 1 & $3 / 2$ & 2 & $5 / 2$ & 3 & $5 / 2$ \\
\hline & 1 & $3 / 2$ & 2 & $3 / 2$ & 1 & $3 / 2$ \\
\hline & 1 & $3 / 2$ & 1 & $3 / 2$ & 1 & $3 / 2$ \\
\hline & 0 & $1 / 2$ & 1 & $3 / 2$ & 1 & $3 / 2$ \\
\hline & 1 & $1 / 2$ & 1 & $3 / 2$ & 1 & $3 / 2$ \\
\hline & 1 & $3 / 2$ & 2 & $5 / 2$ & 2 & $5 / 3$ \\
\hline & 0 & $1 / 2$ & 0 & $1 / 2$ & 1 & $3 / 2$ \\
\hline & 1 & $1 / 2$ & 0 & $1 / 2$ & 1 & $3 / 2$ \\
\hline & 1 & $3 / 2$ & 1 & $1 / 2$ & 1 & $3 / 2$ \\
\hline & 0 & $1 / 2$ & 1 & $1 / 2$ & 1 & $3 / 2$ \\
\hline & 1 & $1 / 2$ & 1 & $1 / 2$ & 1 & $3 / 2$ \\
\hline & 1 & $3 / 2$ & 2 & $3 / 2$ & 2 & $5 / 2$ \\
\hline & 1 & $3 / 2$ & 1 & $3 / 2$ & 2 & $5 / 2$ \\
\hline & 0 & $1 / 2$ & 1 & $3 / 2$ & 2 & $5 / 2$ \\
\hline & 1 & $1 / 2$ & 1 & $3 / 2$ & 2 & $5 / 2$ \\
\hline & 1 & $3 / 2$ & 2 & $5 / 2$ & 3 & $7 / 2$ \\
\hline
\end{tabular}


TABLE III. Energies and rms radii of $N$-nucleon systems interacting via the Malfliet-Tjon potential $\mathrm{V}$ [29]. The value of $\mathcal{K}$ in Tables III-IX denotes the basis dimension beyond which the energies and the radii of the SVM calculation do not change in the digits shown. $\left(\hbar^{2} / m=41.47 \mathrm{MeV}\right.$ $\mathrm{fm}^{2}$ throughout Tables III-VI).

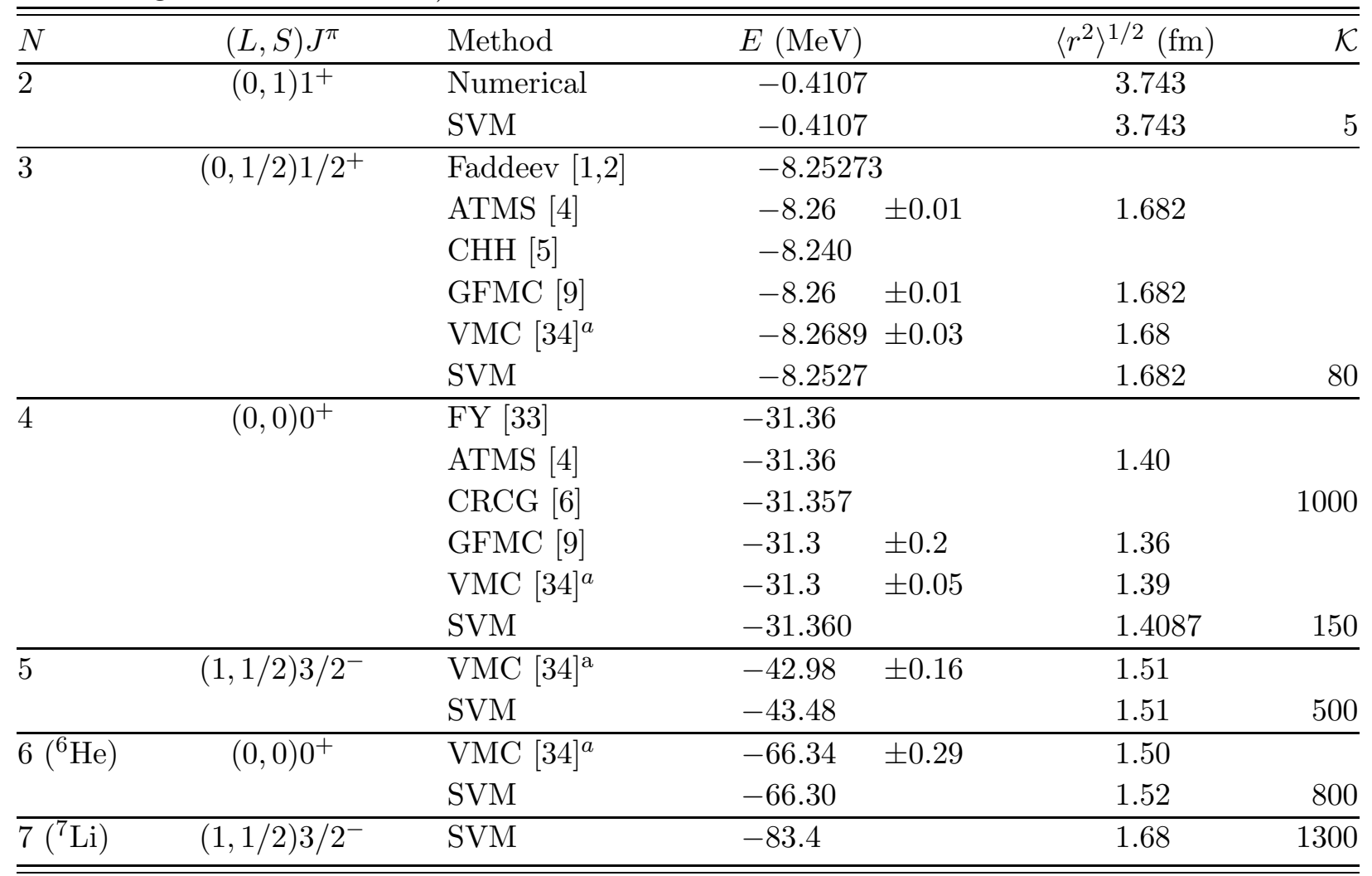

${ }^{a}$ Calculated with Coulomb potential, the Coulomb contribution then subtracted perturbatively. The potential strength used in the VMC [34] calculation is $V_{1}=1458.25$ and $V_{2}=-578.17 \mathrm{MeV}$, which is slightly different from that used in the present calculation. 
TABLE IV. Energies and rms radii of $N$-nucleon systems interacting via the Volkov potential [30]. The Majorana exchange parameter $M$ is set to zero.

\begin{tabular}{|c|c|c|c|c|c|}
\hline $\bar{N}$ & $(L, S) J^{\pi}$ & Method & $E(\mathrm{MeV})$ & $\left\langle r^{2}\right\rangle^{1 / 2}(\mathrm{fm})$ & $\overline{\mathcal{K}}$ \\
\hline \multirow[t]{2}{*}{2} & $(0,1) 1^{+}$ & Numerical & -0.545 & 3.44 & \\
\hline & & SVM & -0.545 & 3.44 & 5 \\
\hline \multirow[t]{4}{*}{$\overline{3}$} & $(0,1 / 2) 1 / 2^{+}$ & Faddeev 33 & -8.43 & & \\
\hline & & Variational 16 & -8.460 & 1.725 & \\
\hline & & $\mathrm{HH} 35$ & -8.4647 & & \\
\hline & & SVM & -8.46 & 1.73 & 30 \\
\hline \multirow[t]{4}{*}{4} & $(0,0) 0^{+}$ & FY 33 & -30.27 & & \\
\hline & & Variational 16] & -29.490 & 1.47 & \\
\hline & & HH 36 & -30.3988 & & \\
\hline & & SVM & -30.42 & 1.49 & 50 \\
\hline 5 & $(1,1 / 2) 3 / 2^{-}$ & SVM & -43.00 & 1.59 & 120 \\
\hline $6\left({ }^{6} \mathrm{Li}\right)$ & $(0,1) 1^{+}$ & SVM & -66.25 & 1.60 & 250 \\
\hline $7\left({ }^{7} \mathrm{Li}\right)$ & $(1,1 / 2) 3 / 2^{-}$ & SVM & -98.75 & 1.57 & $\overline{400}$ \\
\hline
\end{tabular}

TABLE V. Energies and rms radii of $N$-nucleon systems interacting via the Afnan-Tang S3 potential [31].

\begin{tabular}{llllcl}
\hline \hline$N$ & $(L, S) J^{\pi}$ & Method & $E(\mathrm{MeV})$ & $\left\langle r^{2}\right\rangle^{1 / 2}(\mathrm{fm})$ & $\mathcal{K}$ \\
\hline 2 & $(0,1) 1^{+}$ & Numerical & -2.216 & 1.94 & \\
& & SVM & -2.216 & 1.94 & 7 \\
\hline 3 & $(0,1 / 2) 1 / 2^{+}$ & Faddeev [33] & -8.20 & & \\
& & Faddeev [37] & -8.765 & 1.72 & \\
& & $-8.73 \pm 0.10$ & 1.727 & \\
& & GFMC [10] & -6.677 & 1.67 & \\
& & Variational [16] & -8.753 & 1.44 & \\
& & SVM & -28.80 & 1.42 & 140 \\
\hline 4 & $(0,0) 0^{+}$ & FY [33] & -25.654 & 1.48 & 220 \\
& & Variational [16] & -30.37 & 1.49 & 400 \\
\hline 5 & SVM & -44.27 & & \\
\hline $6\left({ }^{6} \mathrm{Li}\right)$ & $(1,1 / 2) 3 / 2^{-}$ & SVM & -70.65 & & \\
\hline \hline
\end{tabular}


TABLE VI. Energies and rms radii of $N$-nucleon systems interacting via the Minnesota potential [32] with the exchange parameter $u=1$. The Coulomb interaction is included $\left(e^{2}=1.44\right.$ $\mathrm{MeV} \mathrm{fm})$. The experimental $\left\langle r^{2}\right\rangle^{1 / 2}$ value is the point charge radius with the proton's finite size corrected.

\begin{tabular}{|c|c|c|c|c|c|}
\hline$N$ & $(L, S) J^{\pi}$ & Method & $E(\mathrm{MeV})$ & $\left\langle r^{2}\right\rangle^{1 / 2}(\mathrm{fm})$ & $\overline{\mathcal{K}}$ \\
\hline \multirow[t]{2}{*}{2} & $(0,1) 1^{+}$ & SVM & -2.202 & 1.952 & 5 \\
\hline & & Exp. & -2.224 & 1.96 & \\
\hline \multirow[t]{2}{*}{$3\left({ }^{3} \mathrm{H}\right)$} & $(0,1 / 2) 1 / 2^{+}$ & SVM & -8.380 & 1.698 & 40 \\
\hline & & Exp. & -8.481 & 1.57 & \\
\hline \multirow[t]{2}{*}{$4\left({ }^{4} \mathrm{He}\right)$} & $(0,0) 0^{+}$ & SVM & -29.937 & 1.41 & 60 \\
\hline & & Exp. & -28.295 & 1.47 & \\
\hline \multirow[t]{2}{*}{5} & $(1,1 / 2) 3 / 2^{-}$ & SVM & unbound & & \\
\hline & & Exp. & unbound & & \\
\hline \multirow[t]{2}{*}{$6\left({ }^{6} \mathrm{He}\right)$} & $(0,0) 0^{+}$ & SVM & -30.07 & 2.44 & 600 \\
\hline & & Exp. & -29.271 & & \\
\hline \multirow[t]{2}{*}{$\left({ }^{6} \mathrm{Li}\right)$} & $(0,1) 1^{+}$ & SVM & -34.59 & 2.22 & 600 \\
\hline & & Exp. & -31.995 & 2.43 & \\
\hline
\end{tabular}

TABLE VII. Energies and rms radii of $N-\alpha$ systems interacting via the Ali-Bodmer potential [39] of Eq. (63). The $\alpha$-particle is considered to be structureless boson. $\left(\hbar^{2} / M_{\alpha}=41.467 / 4 \mathrm{MeV}\right.$ $\left.\mathrm{fm}^{2}\right)$.

\begin{tabular}{lllll}
\hline \hline$N$ & Method & $E(\mathrm{MeV})$ & $\left\langle r^{2}\right\rangle^{1 / 2}(\mathrm{fm})$ & $\mathcal{K}$ \\
\hline 2 & & unbound & & \\
\hline 3 & ATMS [4] & -5.18 & 2.43 & 60 \\
\hline 4 & SVM & -5.18 & 2.43 & \\
& ATMS [4] & -11.1 & 2.65 & 150 \\
& SVM & -11.07 & 2.65 & 400 \\
\hline 5 & SVM & -16.22 & 2.99 & 600 \\
\hline 6 & SVM & -20.13 & 3.32 & \\
\hline \hline
\end{tabular}


TABLE VIII. Energies and rms radii of electron-positron systems treated as fermions (f) and as bosons (b). Atomic units are used.

\begin{tabular}{|c|c|c|c|c|}
\hline System & Method & $E$ & $\left\langle r^{2}\right\rangle^{1 / 2}$ & $\overline{\mathcal{K}}$ \\
\hline \multirow[t]{2}{*}{$\overline{\left(e^{+}, e^{-}\right) \mathrm{b}, \mathrm{f}}$} & SVM & -0.25 & 1.732 & 10 \\
\hline & Exact & -0.25 & 1.732 & \\
\hline \multirow[t]{3}{*}{$\overline{\left(2 e^{+}, e^{-}\right) \mathrm{b}, \mathrm{f}}$} & SVM & -0.262004 & 4.592 & 150 \\
\hline & Variational 41] & -0.2620050702325 & 4.594 & 700 \\
\hline & Faddeev [2] & -0.26202 & & \\
\hline \multirow[t]{2}{*}{$\overline{\left(2 e^{+}, 2 e^{-}\right) \mathrm{b}, \mathrm{f}}$} & SVM & -0.515989 & 3.608 & 300 \\
\hline & Variational 19,46 & -0.515980 & 3.600 & 300 \\
\hline$\overline{\left(3 e^{+}, 2 e^{-}\right) \mathrm{f}}$ & SVM & unbound & & 1000 \\
\hline$\left(3 e^{+}, 2 e^{-}\right) \mathrm{b}$ & SVM & -0.5493 & 3.53 & 200 \\
\hline$\overline{\left(3 e^{+}, 3 e^{-}\right) \mathrm{f}}$ & SVM & unbound & & 1000 \\
\hline \multirow[t]{2}{*}{$\left(3 e^{+}, 3 e^{-}\right) \mathrm{b}$} & SVM & -0.820 & 3.42 & 300 \\
\hline & Variational 44] & -0.789 & & 5 \\
\hline
\end{tabular}

TABLE IX. Energies and rms radii of "self-gravitating" $m$-particle- $n$-antiparticle systems $(m+, n-)$; f: fermions; b: bosons. VLB and VUB stand for the variational lower and upper bounds given in Ref. 49. The units of the energy and length are $G^{2} m^{5} \hbar^{-2}$ and $G^{-1} m^{-3} \hbar^{2}$, respectively.

\begin{tabular}{|c|c|c|c|c|c|}
\hline System & Method & $E$ & & $\left\langle r^{2}\right\rangle^{1 / 2}$ & $\mathcal{K}$ \\
\hline \multirow[t]{2}{*}{$(+,-) b, f$} & SVM & -0.25 & & 1.732 & 10 \\
\hline & Exact & -0.25 & & 1.732 & \\
\hline \multirow{2}{*}{$\overline{(2+,-) b, f}$} & SVM & -1.072 & & 1.304 & 15 \\
\hline & Variational 49 & -1.067 & & & \\
\hline \multirow[t]{2}{*}{$(2+, 2-) b, f$} & SVM & -2.791 & & 1.027 & 100 \\
\hline & VUB (VLB) & -1.951 & $(-3.00)$ & & \\
\hline$\overline{(3+, 2-) \mathrm{f}}$ & SVM & -3.758 & & 1.554 & 200 \\
\hline \multirow[t]{2}{*}{$(3+, 2-) \mathrm{b}$} & SVM & -5.732 & & 0.844 & 200 \\
\hline & VUB (VLB) & -4.336 & $(-6.25)$ & & \\
\hline$\overline{(3+, 3-) \mathrm{f}}$ & SVM & -6.409 & & 1.621 & 300 \\
\hline \multirow[t]{2}{*}{$(3+, 3-) \mathrm{b}$} & SVM & -10.215 & & 0.718 & 300 \\
\hline & VUB (VLB) & -8.130 & $(-11.25)$ & & \\
\hline
\end{tabular}


Table X. Components of the two-particle interaction matrix elements. The operators $B^{p}$ and $C_{l m}^{p}$ are defined in Eq. (70) of Appendix B. The symbol $p$ specifies the component of the two-body interaction. The following additional notations are introduced: $\mathbf{r}_{12}=\mathbf{r}_{1}-\mathbf{r}_{2}$, $\mathbf{p}_{12}=\frac{1}{2 \hbar}\left(\mathbf{p}_{1}-\mathbf{p}_{2}\right), \mathbf{L}=\mathbf{r}_{12} \times \mathbf{p}_{12}, \mathbf{S}=\frac{1}{2}\left(\boldsymbol{\sigma}_{1}+\boldsymbol{\sigma}_{2}\right), S_{12}=3\left(\boldsymbol{\sigma}_{1} \cdot \hat{\mathbf{r}}_{12}\right)\left(\boldsymbol{\sigma}_{2} \cdot \hat{\mathbf{r}}_{12}\right)-\boldsymbol{\sigma}_{1} \cdot \boldsymbol{\sigma}_{2}$, $\mathbf{x}=\mathbf{s}_{1}^{\prime}-\mathbf{s}_{2}^{\prime}$, and $(\mathbf{x})_{m}=\sqrt{\frac{4 \pi}{3}} x Y_{1 m}(\hat{\mathbf{x}})$.

\begin{tabular}{|c|c|c|c|c|c|}
\hline$p$ & definition & $B^{p}$ & $C_{00}^{p}$ & $C_{1 m}^{p}$ & $C_{2 m}^{p}$ \\
\hline \begin{tabular}{|l|}
$c$ \\
\end{tabular} & 1 & 0 & $\sqrt{4 \pi}$ & 0 & 0 \\
\hline$t$ & $S_{12}$ & 0 & 0 & 0 & $\sqrt{\frac{24 \pi}{5}}\left[\boldsymbol{\sigma}_{1} \times \boldsymbol{\sigma}_{2}\right]_{m}^{(2)}$ \\
\hline $\mathrm{b}$ & $\mathbf{L} \cdot \mathbf{S}$ & 0 & 0 & $-\sqrt{\frac{2 \pi}{3}} \nu\left[\mathbf{x} \times\left(\boldsymbol{\sigma}_{1}+\boldsymbol{\sigma}_{2}\right)\right]_{m}^{(1)}$ & 0 \\
\hline$q$ & $\mathbf{L}^{2}$ & $-\frac{2}{3} \nu^{2} \mathbf{x}^{2}$ & 0 & $\sqrt{\frac{16 \pi}{3}} \nu(\mathbf{x})_{m}$ & $\sqrt{\frac{8 \pi}{15}} \nu^{2}[\mathbf{x} \times \mathbf{x}]_{m}^{(2)}$ \\
\hline$q$ & $\mathbf{p}_{12}^{2}$ & $\nu^{2}$ & $\sqrt{4 \pi} \nu\left(-3+\nu \mathbf{x}^{2}\right)$ & $-\sqrt{\frac{16 \pi}{3}} \nu^{2}(\mathbf{x})_{m}$ & 0 \\
\hline$b b$ & $(\mathbf{L} \cdot \mathbf{S})^{2}$ & $\begin{array}{c}-\frac{1}{6} \nu^{2}\left\{\mathbf{x}^{2}\left(2+\boldsymbol{\sigma}_{1} \cdot \boldsymbol{\sigma}_{2}\right)-\right. \\
\left.\left(\mathbf{x} \cdot \boldsymbol{\sigma}_{1}\right)\left(\mathbf{x} \cdot \boldsymbol{\sigma}_{2}\right)\right\}\end{array}$ & 0 & $\begin{array}{c}\sqrt{\frac{\pi}{3}} \nu\left\{(\mathbf{x})_{m}\left(2+\boldsymbol{\sigma}_{1} \cdot \boldsymbol{\sigma}_{2}\right)+\right. \\
\frac{1}{\sqrt{2}}\left[\mathbf{x} \times\left(\boldsymbol{\sigma}_{1}+\boldsymbol{\sigma}_{2}\right)\right]_{m}^{(1)} \\
\left.-\frac{1}{2}\left(\boldsymbol{\sigma}_{1 m}\left(\mathbf{x} \cdot \boldsymbol{\sigma}_{2}\right)+\boldsymbol{\sigma}_{2 m}\left(\mathbf{x} \cdot \boldsymbol{\sigma}_{1}\right)\right)\right\}\end{array}$ & $\begin{array}{c}\sqrt{\frac{2 \pi}{15}} \nu^{2}\left\{[\mathbf{x} \times \mathbf{x}]_{m}^{(2)}+\right. \\
\left.2\left[\left[\mathbf{x} \times \boldsymbol{\sigma}_{1}\right]^{(1)} \times\left[\mathbf{x} \times \boldsymbol{\sigma}_{2}\right]^{(1)}\right]_{m}^{(2)}\right\}\end{array}$ \\
\hline
\end{tabular}




\section{Figure Captions}

Fig. 1 : Different sets of relative coordinates for a system of six identical particles.

Fig. 2 : Convergence of the ${ }^{6} \mathrm{Li}$ energy on different random paths. The Volkov potential [30] is used.

Fig. 3 : Convergence of the $\alpha$-particle energy for the potentials listed in Table I. 


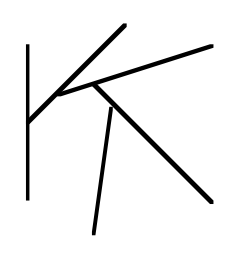

1 a

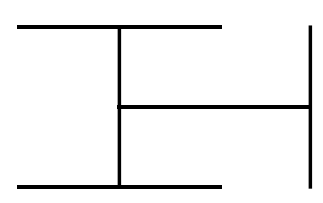

$1 d$

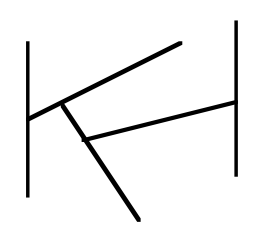

$1 \mathrm{~b}$

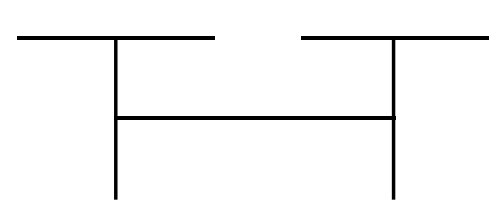

1 e

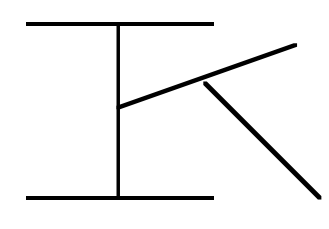

$1 \mathrm{c}$

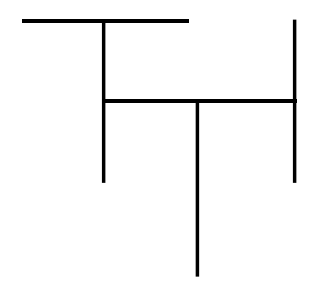

$1 \mathrm{f}$ 


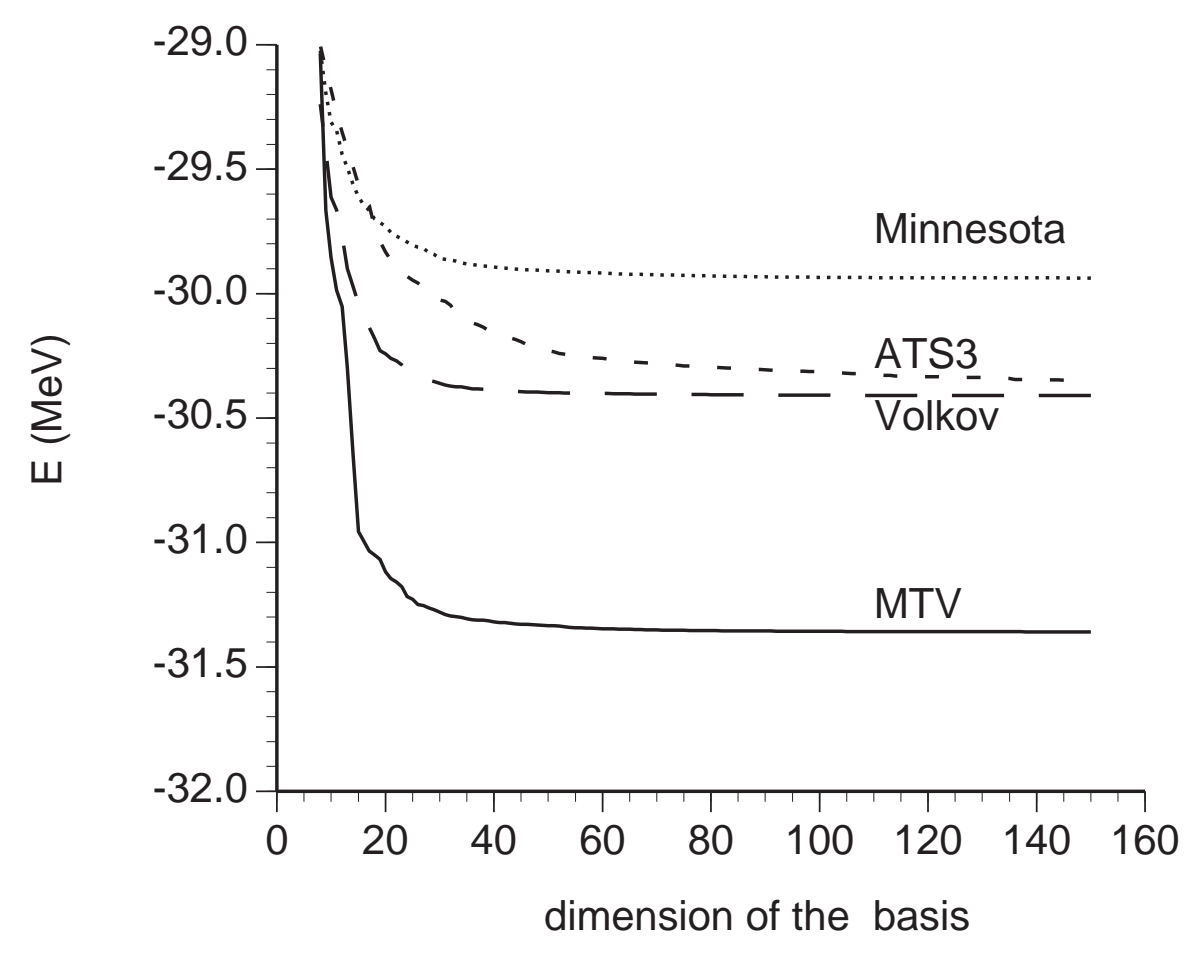




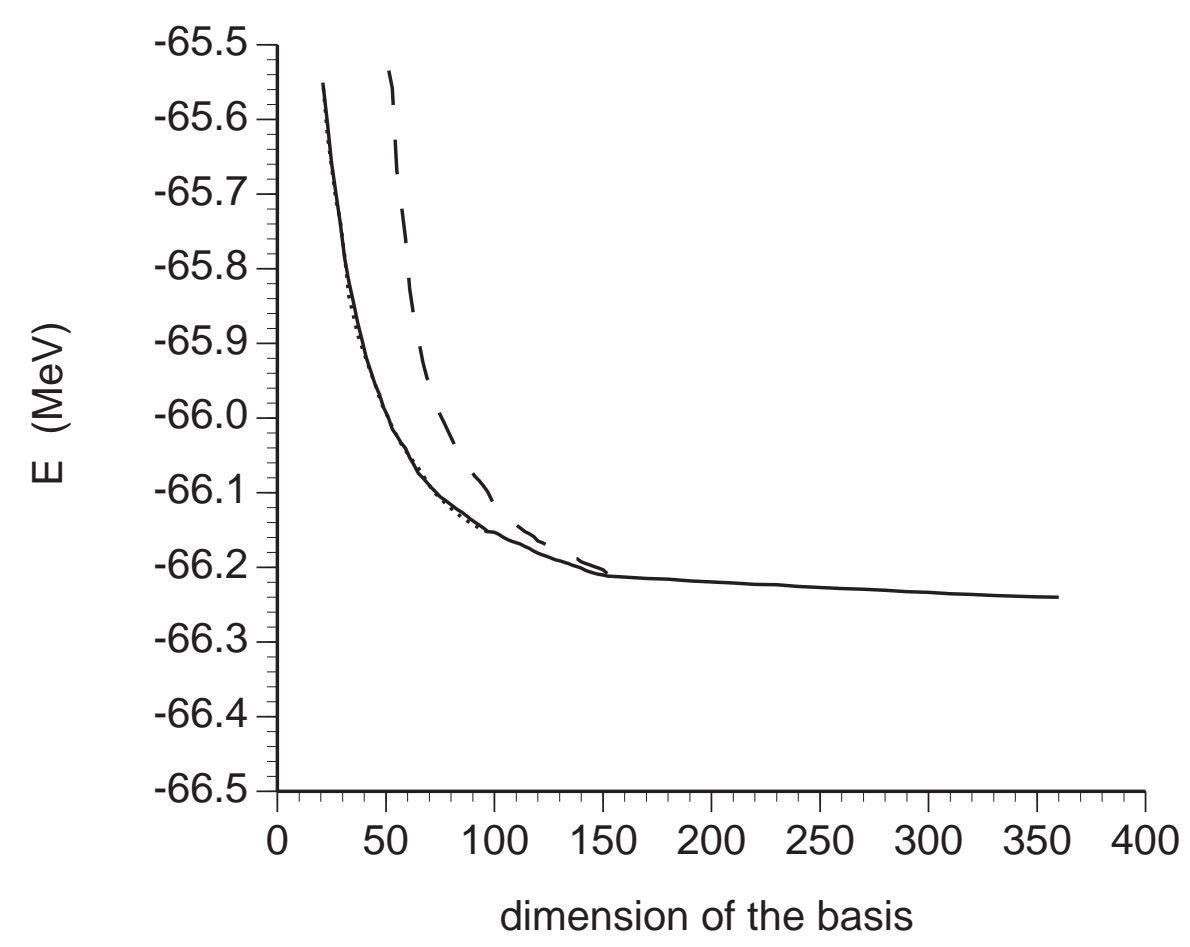

